\title{
Essential dimension of extensions of finite groups by tori
}

\author{
Zinovy Reichstein and Federico Scavia
}

\begin{abstract}
Let $p$ be a prime, $k$ be a $p$-closed field of characteristic different from $p$, and $1 \rightarrow T \rightarrow$ $G \rightarrow F \rightarrow 1$ be an exact sequence of algebraic groups over $k$, where $T$ is a torus and $F$ is a finite $p$-group. In this paper, we study the essential dimension $\operatorname{ed}(G ; p)$ of $G$ at $p$. R. Lötscher, M. MacDonald, A. Meyer, and the first author showed that

$$
\min \operatorname{dim}(V)-\operatorname{dim}(G) \leqslant \operatorname{ed}(G ; p) \leqslant \min \operatorname{dim}(W)-\operatorname{dim}(G),
$$

where $V$ and $W$ range over the $p$-faithful and $p$-generically free $k$-representations of $G$, respectively. In the special case where $G=F$, one recovers the formula for $\operatorname{ed}(F ; p)$ proved earlier by N. Karpenko and A. Merkurjev. In the case where $F=T$, one recovers the formula for ed $(T ; p)$ proved earlier by R. Lötscher et al. In both of these cases, the upper and lower bounds on $\operatorname{ed}(G ; p)$ given above coincide. In general, there is a gap between them. Lötscher et al. conjectured that the upper bound is, in fact, sharp; that is, $\operatorname{ed}(G ; p)=\min \operatorname{dim}(W)-\operatorname{dim}(G)$, where $W$ ranges over the $p$-generically free representations. We prove this conjecture in the case where $F$ is diagonalizable.
\end{abstract}

\section{Introduction}

Let $p$ be a prime integer and $k$ be a $p$-closed field of characteristic different from $p$. That is, the degree of every finite extension $l / k$ is a power of $p$. Consider an algebraic group $G$ defined over $k$ which fits into the exact sequence

$$
1 \longrightarrow T \longrightarrow G \stackrel{\pi}{\longrightarrow} F \longrightarrow 1,
$$

where $T$ is a (not necessarily split) torus and $F$ is a (not necessarily constant) finite $p$-group defined over $k$. We say that a linear representation $G \rightarrow \mathrm{GL}(V)$ is $p$-faithful if its kernel is a finite subgroup of $G$ of order prime to $p$ and $p$-generically free if the isotropy subgroup $G_{v}$ is a finite group of order prime to $p$ for $v \in V(\bar{k})$ in general position. We denote by $\eta(G)$ (respectively, $\rho(G)$ ) the smallest dimension of a $p$-faithful (respectively, $p$-generically free) representation of $G$ defined over $k$. R. Lötscher, M. MacDonald, A. Meyer, and the first author [LMMR13b, Theorem 1.1]

Received 30 March 2020, accepted in final form 12 April 2021.

2020 Mathematics Subject Classification 20G15, 14L30, $14 \mathrm{E} 05$.

Keywords: essential dimension, algebraic torus, stabilizer in general position.

This journal is (C) Foundation Compositio Mathematica 2021. This article is distributed with Open Access under the terms of the Creative Commons Attribution Non-Commercial License, which permits non-commercial reuse, distribution, and reproduction in any medium, provided that the original work is properly cited. For commercial re-use, please contact the Foundation Compositio Mathematica.

Zinovy Reichstein was partially supported by National Sciences and Engineering Research Council of Canada Discovery grant 253424-2017. Federico Scavia was partially supported by a graduate fellowship from the University of British Columbia. 


\section{Z. Reichstein and F. Scavia}

have shown that the essential $p$-dimension $\operatorname{ed}(G ; p)$ of $G$ over $k$ satisfies the inequalities

$$
\eta(G)-\operatorname{dim}(G) \leqslant \operatorname{ed}(G ; p) \leqslant \rho(G)-\operatorname{dim}(G) .
$$

For the definition of $\operatorname{ed}(G ; p)$, see Section 4 .

The inequalities (1.2) represent a common generalization of the formulas for the essential $p$-dimension of a finite constant $p$-group, due to N. Karpenko and A. Merkurjev [KM08, Theorem 4.1] (where $T=\{1\}$ ), and of an algebraic torus, due to R. Lötscher et al. [LMMR13a] (where $F=\{1\}$ ). In both of these cases, every $p$-faithful representation of $G$ is $p$-generically free, and thus $\eta(G)=\rho(G)$. In general, $\eta(G)$ can be strictly smaller than $\rho(G)$. Lötscher et al. conjectured that the upper bound of (1.2) is, in fact, sharp.

Conjecture 1.1. Let $p$ be a prime integer, $k$ be a $p$-closed field of characteristic different from $p$, and $G$ be an affine algebraic group defined over $k$. Assume that the connected component $G^{0}=T$ is a $k$-torus and the component group $G / G^{0}=F$ is a finite $p$-group. Then

$$
\operatorname{ed}(G ; p)=\rho(G)-\operatorname{dim} G,
$$

where $\rho(G)$ is the minimal dimension of a $p$-generically free $k$-representation of $G$.

Informally speaking, the lower bound of $(1.2)$ is the strongest lower bound on $\operatorname{ed}(G ; p)$ one can hope to prove by the methods of [KM08, LMMR13a] and [LMMR13b]. In the case where the upper and lower bounds of (1.2) diverge, Conjecture 1.1 calls for a new approach.

Conjecture 1.1 appeared in print in [Rei10, Section 7.9] on the list of open problems in the theory of essential dimension. The only bit of progress since then has been a proof in the special case where $G$ is a semi-direct product of a cyclic group $F=\mathbb{Z} / p \mathbb{Z}$ of order $p$ and a split torus $T=\mathbb{G}_{\mathrm{m}}^{n}$, due to M. Huruguen (unpublished). Huruguen's argument relies on the classification of integral representations of $\mathbb{Z} / p \mathbb{Z}$ due to F. Diederichsen and I. Reiner [CR62, Theorem 74.3]. So far, this approach has resisted all attempts to generalize it beyond the case where $G \simeq \mathbb{G}_{\mathrm{m}}^{n} \rtimes(\mathbb{Z} / p \mathbb{Z})$.

Note that $\eta(G)$ is often accessible by cohomological and/or combinatorial techniques; see Section 6 and Lemma 9.3, as well as the remarks after this lemma. Computing $\rho(G)$ is usually a more challenging problem. The purpose of this paper is to establish Conjecture 1.1 in the case where $F$ is a diagonalizable abelian $p$-group. Moreover, our main result also gives a way of computing $\rho(G)$ in this case.

THEOREM 1.2. Let $p$ be a prime integer, $k$ be a $p$-closed field of characteristic different from $p$, and $G$ be an extension of a (not necessarily constant) diagonalizable p-group $F$ by a (not necessarily split) torus $T$, as in (1.1).

(a) We have $\operatorname{ed}(G ; p)=\rho(G)-\operatorname{dim} G$.

(b) Moreover, suppose that $V$ is a $p$-faithful representation of $G$ of minimal dimension, $\bar{k}$ is the algebraic closure of $k$, and $S_{V} \subset G_{\bar{k}}$ is a stabilizer in general position for the $G_{\bar{k}}$-action on $V_{\bar{k}}$. Then $\rho(G)=\eta(G)+\operatorname{rank}_{p}\left(S_{V}\right)$.

Here $\operatorname{rank}_{p}\left(S_{V}\right)$ is the largest $r$ such that $S_{V}$ contains a subgroup isomorphic to $\mu_{p}^{r}$. Note that $S_{V}$ exists by Lemma 2.1. Most of the remainder of this paper (Sections 2-8) will be devoted to proving Theorem 1.2. A key ingredient in the proof is the resolution theorem (Theorem 7.2), which is based, in turn, on an old valuation-theoretic result of M. Artin and O. Zariski [Art86, Theorem 5.2]. In Section 9, we will use Theorem 1.2 to complete the computation of $\operatorname{ed}(N ; p)$ initiated in [MR09] and [Mac11]. Here $N$ is the normalizer of a split maximal torus in a split simple algebraic group. 


\section{EXTENSIONS OF FINITE GROUPS BY TORI}

\section{Stabilizers in general position}

In this section, we assume that the base field $k$ is algebraically closed. Let $G$ be a linear algebraic group defined over $k$. A $G$-variety $X$ is called primitive if $G$ transitively permutes the irreducible components of $X$.

Let $X$ be a primitive $G$-variety. A subgroup $S \subset G$ is called a stabilizer in general position for the $G$-action on $X$ if there exists an open $G$-invariant subset $U \subset X$ such that $\operatorname{Stab}_{G}(x)$ is conjugate to $S$ for every $x \in U(k)$. Note that a stabilizer in general position does not always exist. See [PV94, Example 7.1.1] for an easy example where $G$ is unipotent; further examples, with $G=\mathrm{SL}_{n}$, can be found in [Ric72, Section 12.4]. When a stabilizer in general position $S \subset G$ exists, it is unique up to conjugacy.

Lemma 2.1. Let $G$ be a linear algebraic group over $k$ and $X$ be a primitive quasi-projective $G$-variety. Assume that the connected component $T=G^{0}$ is a torus and the component group $F=G / G^{0}$ is finite of order prime to $\operatorname{char}(k)$. Then there exists a stabilizer in general position $S \subset G$.

Proof. After replacing $G$ with $\bar{G}:=G /(K \cap T)$, where $K$ is the kernel of the $G$-action on $X$, we may assume that the $T$-action on $X$ is faithful and, hence, generically free. In other words, for $x \in X(k)$ in general position, $\operatorname{Stab}_{G}(x) \cap T=1$; in particular, $\operatorname{Stab}_{G}(x)$ is a finite $p$-group. Since $\operatorname{char}(k) \neq p$, Maschke's theorem tells us that $\operatorname{Stab}_{G}(x)$ is linearly reductive. Hence, for $x \in X(k)$ in general position, $\operatorname{Stab}_{G}(x)$ is $G$-completely reducible; see [Jan04, Lemma 11.24]. The lemma now follows from [Mar15, Corollary 1.5].

Remark 2.2. The condition that $X$ is quasi-projective can be dropped if $k=\mathbb{C}$; see [Ric72, Theorem 9.3.1]. With a bit more effort, this condition can also be removed for any algebraically closed base field $k$ of characteristic different from $p$. Since we shall not need this more general variant of Lemma 2.1, we leave its proof as an exercise for the reader.

We define the (geometric) $p$-rank $\operatorname{rank}_{p}(G)$ of an algebraic group $G$ to be the largest integer $r$ such that $G$ contains a subgroup isomorphic to $\mu_{p}^{r}=\mu_{p} \times \cdots \times \mu_{p}(r$ times $)$.

Lemma 2.3. Let $X$ be a normal $G$-variety and $Y \subset X$ be a $G$-invariant prime divisor of $X$. Let $S_{X}$ and $S_{Y}$ be stabilizers in general position of the $G$-actions on $X$ and $Y$, respectively. Assume that $p$ is a prime and $\operatorname{char}(k) \neq p$.

(a) We have $\operatorname{rank}_{p}\left(S_{Y}\right) \leqslant \operatorname{rank}_{p}\left(S_{X}\right)+1$.

(b) Assume that the $G$-action on $X$ is $p$-faithful. Denote the kernel of the $G$-action on $Y$ by $N$. Then there is a group homomorphism $\alpha: N \rightarrow \mathbb{G}_{\mathrm{m}}$ such that $\operatorname{Ker}(\alpha)$ does not contain a subgroup of order $p$.

Proof. Let $U \subset X$ be a $G$-invariant dense open subset of $X$ such that $\operatorname{Stab}_{G}(x)$ is conjugate to $S_{X}$ for every $x \in U(k)$. If $Y \cap U \neq \emptyset$, then $S_{Y}=S_{X}$, and we are done. Thus we may assume that $Y$ is contained in $Z=X \backslash U$. Since $Y$ is a prime divisor in $X$, it is an irreducible component of $Z$. After removing all other irreducible components of $Z$ from $X$, we may assume that $Z=Y$. Since $X$ is normal, $Y$ intersects the smooth locus of $X$ non-trivially. Choose a $k$-point $y \in Y$ such that both $X$ and $Y$ are smooth at $y$ and $\operatorname{Stab}_{G}(y)$ is conjugate to $S_{Y}$. After replacing $S_{Y}$ with a conjugate, we may assume that $\operatorname{Stab}_{G}(y)=S_{Y}$. The group $\operatorname{Stab}_{G}(y)$ acts on the tangent spaces $T_{y}(X)$ and $T_{y}(Y)$, hence on the 1-dimensional normal space $T_{y}(X) / T_{y}(Y)$. This gives rise to a character $\alpha: S_{Y} \rightarrow \mathbb{G}_{\mathrm{m}}$. 


\section{Z. Reichstein and F. Scavia}

(a) Assume the contrary: $S_{Y}$ contains $\mu_{p}^{r+2}$, where $r=\operatorname{rank}_{p}\left(S_{X}\right)$. Then the kernel of $\alpha$ contains a subgroup $\mu \simeq \mu_{p}^{r+1}$. By Maschke's theorem, the natural projection $T_{y}(X) \rightarrow T_{y}(X) / T_{y}(Y)$ is $\mu$-equivariantly split. Equivalently, there exists a $\mu$-invariant tangent vector $v \in T_{y}(X)$ which does not belong to $T_{y}(Y)$. By the Luna slice theorem,

$$
T_{y}(X)^{\mu}=T_{y}\left(X^{\mu}\right) .
$$

For a proof in characteristic 0, see [PV94, Section 6.5]. Generally speaking, Luna's theorem fails in prime characteristic, but (2.1) remains valid because $\mu$ is linearly reductive; see [BR85, Lemma 8.3]. Now observe that since $\mu$ does not fit into any conjugate of $S_{X}$, the subvariety $X^{\mu}$ is contained in $Y=X \backslash U$. Thus $v \in T_{y}(X)^{\mu}=T_{y}\left(X^{\mu}\right) \subset T_{y}(Y)$, which gives a contradiction.

(b) Let $y \in Y$ be a smooth $k$-point of $X$ and $Y$, and $S_{y}=\operatorname{Stab}_{G}(y)$ as in part (a). Then $N$ is contained in $S_{Y}$, and $\alpha$ restricts to a character $N \rightarrow \mathbb{G}_{\mathrm{m}}$. It suffices to show that the kernel of $\alpha$ in $S_{Y}$ does not contain a subgroup of order $p$. Assume the contrary: a subgroup $H$ of order $p$ lies in the kernel of $\alpha$. Then $H$ fixes a smooth point $y$ of $X$ and acts trivially on both $T_{y}(Y)$ and $T_{y}(X) / T_{y}(Y)$ and hence (since $H$ is linearly reductive) on $T_{y}(X)$. It is well known that this implies that $H$ acts trivially on $X$; see, for example, the proof of [GR09, Lemma 4.1]. This contradicts our assumption that the $G$-action on $X$ is $p$-faithful.

\section{Covers}

Let $k$ be an arbitrary field, and let $G$ be a linear algebraic group defined over $k$. As usual, we will denote the algebraic closure of $k$ by $\bar{k}$. A $G$-variety $X$ is called primitive if the $G_{\bar{k}}$-variety $X_{\bar{k}}$ is primitive. A dominant $G$-equivariant rational map $X \rightarrow Y$ of primitive $G$-varieties is called a cover of degree $d$ if $[k(X): k(Y)]=d$. Here if $X_{1}, \ldots, X_{n}$ are the irreducible components of $X$, then $k(X)$ is defined as $k\left(X_{1}\right) \oplus \cdots \oplus k\left(X_{n}\right)$.

Lemma 3.1. Let $p$ be a prime integer, $G$ be a smooth algebraic group such that $G / G^{0}$ is a finite $p$-group, $W$ be an irreducible $G$-variety, $Z \subset W$ be an irreducible $G$-invariant divisor in $W$, and $\tau: X \rightarrow W$ be a $G$-equivariant cover of degree prime to $p$. Then there exists a commutative diagram of $G$-equivariant maps

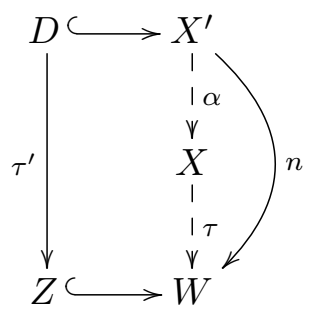

such that $X^{\prime}$ is normal, $\alpha$ is a birational isomorphism, $D$ is an irreducible divisor in $X^{\prime}$, and $\tau^{\prime}$ is a cover of $Z$ of degree prime to $p$.

Proof. Let $X^{\prime}$ be the normalization of $W$ in the function field $k(X)$. Since $G$ acts compatibly on $W$ and $X$, there is a $G$-action on $X^{\prime}$ such that the normalization map $n: X^{\prime} \rightarrow W$ is $G$ equivariant. Over the dense open subset of $W$ where $\tau$ is finite, $n$ factors through $X$. Thus $n$ factors into a composition of a birational isomorphism $\alpha: X^{\prime} \rightarrow X$ and $\tau: X \rightarrow W$. This gives us the right column in the diagram.

To construct $D$, we argue as in the proof of [RY00, Proposition A.4]. Denote the irreducible components of the preimage of $Z$ under $n$ by $D_{1}, \ldots, D_{r} \subset X^{\prime}$. These components are permuted by $G$. Denote the orbits of this permutation action by $\mathcal{O}_{1}, \ldots, \mathcal{O}_{m}$. After renumbering $D_{1}, \ldots, D_{r}$, 


\section{EXTENSIONS OF FINITE GROUPS BY TORI}

we may assume that $D_{i} \in \mathcal{O}_{i}$ for $i=1, \ldots, m$. By the ramification formula (see, for example, [Lan02, XII, Corollary 6.3]),

$$
d=\sum_{i=1}^{m}\left|\mathcal{O}_{i}\right| \cdot\left[D_{i}: Z\right] \cdot e_{i},
$$

where $\left[D_{i}: Z\right]$ denotes the degree of the cover $n_{\mid D_{i}}: D_{i} \rightarrow Z$ and $e_{i}$ is the ramification index of $n$ at the generic point of $D_{i}$. Since $d$ is prime to $p$ and each $\left|\mathcal{O}_{i}\right|$ is a power of $p$, we conclude that there exists an $i \in\{1, \ldots, m\}$ such that $\left|\mathcal{O}_{i}\right|=1$ (that is, $D_{i}$ is $G$-invariant) and $\left[D_{i}: Z\right]$ is prime to $p$. We now set $D=D_{i}$ and $\tau^{\prime}=n_{\mid D_{i}}$.

Lemma 3.2. Let $G$ be a linear algebraic group over an algebraically closed field $k, p \neq \operatorname{char}(k)$ be a prime number, and $\tau: X \rightarrow W$ be a cover of $G$-varieties of degree $d$. Assume that stabilizers in general position for the $G$-actions on $X$ and $W$ exist; denote them by $S_{X}$ and $S_{W}$, respectively. Assume that $d$ is prime to $p$.

(a) If $H$ is a finite $p$-subgroup of $S_{W}$, then $S_{X}$ contains a conjugate of $H$.

(b) We have $\operatorname{rank}_{p}\left(S_{X}\right)=\operatorname{rank}_{p}\left(S_{W}\right)$.

Proof. (a) After replacing $W$ with a dense open subvariety, we may assume that the stabilizer of every point in $W$ is a conjugate of $S_{W}$. Furthermore, after replacing $X$ with the normal closure of $W$ in $k(X)$, we may assume that $\tau$ is a finite morphism. We claim that $W^{S_{W}} \subset \tau\left(X^{H}\right)$. Indeed, suppose $w \in W^{S_{W}}$. Then $H$ acts on $\tau^{-1}(w)$, which is a zero-cycle on $X$ of degree $d$. Since $H$ is a $p$-group, it fixes a $k$-point in $\tau^{-1}(w)$. Hence, $X^{H} \cap \tau^{-1}(w) \neq \emptyset$ or, equivalently, $w \in \tau\left(X^{H}\right)$. This proves the claim.

Since the stabilizer of every point of $W$ is conjugate to $S_{W}$, we have $G \cdot W^{S_{W}}=W$. By the claim, $\tau\left(G \cdot X^{H}\right)=G \cdot \tau\left(X^{H}\right)=W$. Since $G$ acts transitively on the irreducible components of $X$, this implies that $G \cdot X^{H}$ contains a dense open subset $X_{0} \subset X$. In other words, the stabilizer of every point of $X_{0}$ contains a conjugate of $H$, and part (a) follows.

(b) Clearly $S_{X} \subset S_{W}$ and thus $\operatorname{rank}_{p}\left(S_{X}\right) \leqslant \operatorname{rank}_{p}\left(S_{W}\right)$. On the other hand, if $S_{W}$ contains $H=\mu_{p}^{r}$ for some $r \geqslant 0$, then by part (a), the group $S_{X}$ also contains a copy of $\mu_{p}^{r}$. This proves the opposite inequality, $\operatorname{rank}_{p}\left(S_{X}\right) \geqslant \operatorname{rank}_{p}\left(S_{W}\right)$.

\section{Essential $p$-dimension}

Let $X$ and $Y$ be $G$-varieties. Assume that $X$ is primitive. By a $G$-equivariant correspondence $X \rightsquigarrow Y$ of degree $d$, we mean a diagram of rational maps

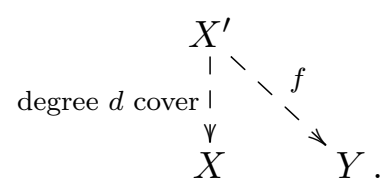

Here we require $X^{\prime}$ to be primitive. We say that this correspondence is dominant if $f$ is dominant. A rational map may be viewed as a correspondence of degree 1 .

The essential dimension $\operatorname{ed}(X)$ of a generically free $G$-variety $X$ is the minimal value of $\operatorname{dim}(Y)-\operatorname{dim}(G)$, where the minimum is taken over all generically free $G$-varieties $Y$ admitting a dominant rational map $X \rightarrow Y$. For a prime integer $p$, the essential dimension $\operatorname{ed}(X ; p)$ of $X$ at $p$ is defined in a similar manner, as $\operatorname{dim}(Y)-\operatorname{dim}(G)$, where the minimum is taken over all generically free $G$-varieties $X$ admitting a $G$-equivariant dominant correspondence $X \rightsquigarrow Y$ of 


\section{Z. Reichstein And F. SCAVia}

degree prime to $p$. Note that these numbers depend on the base field $k$, which we assume to be fixed throughout.

It follows from [LMMR13b, Propositions 2.4 and 3.1] that this minimum does not change if we allow the $G$-action on $Y$ to be $p$-generically free rather than generically free; we shall not need this fact. We will, however, need the following lemma.

Lemma 4.1. Requiring $Y$ to be projective in the above definitions does not change the values of $\operatorname{ed}(X)$ and $\operatorname{ed}(X ; p)$. That is, for any primitive generically free $G$-variety $X$,

(a) there exists a $G$-equivariant dominant rational map $X \rightarrow Z$ where $Z$ is projective, the $G$-action on $Z$ is generically free, and $\operatorname{dim}(Z)=\operatorname{ed}(X ; G)+\operatorname{dim}(G)$;

(b) there exists a $G$-equivariant dominant correspondence $X \rightsquigarrow Z^{\prime}$ of degree prime to $p$ where $Z^{\prime}$ is projective, the $G$-action on $Z^{\prime}$ is generically free, and $\operatorname{dim}\left(Z^{\prime}\right)=\operatorname{ed}(X ; p)+\operatorname{dim}(G)$.

Proof. Let $Y$ be a generically free $G$-variety and $V$ be a generically free linear representation of $G$. It is well known that the $G$-action on $V$ is versal; see, for example, [Mer13, Proposition 3.10]. Consequently, there exist a $G$-invariant subvariety $Y_{1} \subset V$ and a $G$-equivariant dominant rational map $Y \rightarrow Y_{1}$ such that the $G$-action on $Y_{1}$ is generically free. After replacing $Y_{1}$ with its Zariski closure $Z$ in $\mathbb{P}(V \oplus k)$, where $G$ acts trivially on $k$, we obtain a $G$-equivariant dominant rational map $\alpha: Y \rightarrow Z$ such that $Z$ is projective and the $G$-action on $Z$ is generically free.

To prove part (a), choose a dominant $G$-equivariant rational map $f: X \rightarrow Y$ such that the $G$-action on $Y$ is generically free and $\operatorname{dim}(Y)$ is the smallest possible, that is, $\operatorname{dim}(Y)=$ $\operatorname{ed}(X)+\operatorname{dim}(G)$. Now compose $f$ with the map $\alpha: Y \rightarrow Z$ constructed above. By the minimality of $\operatorname{dim}(Y)$, we have $\operatorname{dim}(Z)=\operatorname{dim}(Y)$, and part (a) follows. The proof of part (b) is the same, except that the rational map $f$ is replaced by a correspondence of degree prime to $p$.

The essential dimension $\operatorname{ed}(G)$ (respectively, the essential dimension $\operatorname{ed}(G ; p)$ at $p$ ) of the group $G$ is the maximal value of $\operatorname{ed}(X)$ (respectively, of $\operatorname{ed}(X ; p)$ ) taken over all generically free $G$-varieties $X$.

\section{The groups $G_{n}$}

Let $G$ be an algebraic group over $k$ such that the connected component $T=G^{0}$ is a torus and the component group $F=G / T$ is a finite $p$-group, as in (1.1). By [LMMR13b, Lemma 5.3], there exists a finite $p$-subgroup $F^{\prime} \subset G$ such that $\left.\pi\right|_{F^{\prime}}: F^{\prime} \rightarrow F$ is surjective. We will refer to $F^{\prime}$ as a "quasi-splitting subgroup" for $G$. We will denote the subgroup generated by $F^{\prime}$ and $T[n]$ by $G_{n}$. Here $T[n]$ denotes the $n$-torsion subgroup of $T$, that is, the kernel of the homomorphism $T \stackrel{\times n}{\longrightarrow} T$. Note that our definition of $G_{n}$ depends on the choice of the quasisplitting subgroup $F^{\prime}$. We will assume that $F^{\prime}$ is fixed throughout. We will be particularly interested in the subgroups

$$
G_{1} \subset G_{p} \subset G_{p^{2}} \subset G_{p^{3}} \subset \cdots .
$$

Informally speaking, we will show that these groups approximate " $p$-primary behavior" of $G$ in various ways; see Lemma 5.2 and Proposition 6.2(b) below.

From here on, we denote the center of $G$ by $Z(G)$.

Lemma 5.1. (a) Let $z \in Z(G)(\bar{k})$ be a central element of $G$ of order $p^{n}$ for some $n \geqslant 0$. Then $z \in G_{p^{m}}(\bar{k})$ for $m \gg 0$. 


\section{EXTENSIONS OF FINITE GROUPS BY TORI}

(b) For every $n \geqslant 0$, we have $Z(G)\left[p^{n}\right]=Z\left(G_{p^{r}}\right)\left[p^{n}\right]$ as group schemes for all $r \gg 0$.

Proof. (a) By the definition of $F^{\prime}$, there exist a $g \in F^{\prime}(\bar{k})$ and a $t \in T(\bar{k})$ such that $g=z t$. Since $F^{\prime}$ is a $p$-group, $g^{N}=1$, where $N$ is a sufficiently high power of $p$. Taking $N \geqslant p^{n}$, we also have $z^{N}=1$. Since $z$ is central, $1=g^{N}=(z t)^{N}=z^{N} t^{N}=t^{N}$. Thus $t \in T[N](\bar{k}) \subset G_{N}(\bar{k})$, and, consequently, $z=g t^{-1}$ is a $\bar{k}$-point of $F^{\prime} \cdot T[N]=G_{N}$.

(b) Let $n \geqslant 0$ be fixed. Since both $Z(G)\left[p^{n}\right]$ and $G_{p^{r}}$ are finite $p$-groups and we are assuming that $\operatorname{char}(k) \neq p$, part (a) tells us that there exists an $m \geqslant 0$ such that $Z(G)\left[p^{n}\right] \subset Z\left(G_{p^{r}}\right)\left[p^{n}\right]$ as group schemes for all $r \geqslant m$.

Let $r \geqslant 0$, and let $x \in Z\left(G_{p^{r}}\right)\left[p^{n}\right](\bar{k})$. Let $f_{x}: T_{\bar{k}} \rightarrow T_{\bar{k}}$ be the homomorphism of conjugation by $x$. Passing to character lattices, we obtain a homomorphism $\langle x\rangle \rightarrow \mathrm{GL}_{d}(\mathbb{Z})$, where $d=\operatorname{rank} X\left(T_{\bar{k}}\right)$. By a theorem of Jordan, in $\mathrm{GL}_{d}(\mathbb{Z})$ there are at most finitely many finite subgroups up to conjugacy. In particular, we may find an integer $N \gg 0$ such that the restriction of $\mathrm{GL}_{d}(\mathbb{Z}) \rightarrow \mathrm{GL}_{d}\left(\mathbb{Z} / p^{N} \mathbb{Z}\right)$ to every finite subgroup is injective.

Thus, if $r \geqslant N$, then $f_{x}$ is the identity for every $x \in Z\left(G_{p^{r}}\right)\left[p^{n}\right](\bar{k})$. Since $F^{\prime}$ is contained in $G_{p^{r}}$, every $x \in Z\left(G_{p^{r}}\right)\left[p^{n}\right](\bar{k})$ commutes with $F^{\prime}$. Since $G^{0}$ and $F^{\prime}$ generate $G$, we deduce that $x \in Z(G)\left[p^{n}\right](\bar{k})$. This shows that $Z\left(G_{p^{r}}\right)\left[p^{n}\right] \subset Z(G)\left[p^{n}\right]$ for $r \geqslant N$. We conclude that for $r \geqslant \max (N, m)$, we have $Z\left(G_{p^{r}}\right)\left[p^{n}\right]=Z(G)\left[p^{n}\right]$.

Lemma 5.2. Let $K$ be a $p$-closed field containing $k$. Then every class $\alpha \in H^{1}(K, G)$ lies in the image of the map $H^{1}\left(K, G_{p^{r}}\right) \rightarrow H^{1}(K, G)$ for sufficiently high $r$.

Proof. Let $\alpha \in H^{1}(K, G)$. Consider the commutative diagram with exact rows

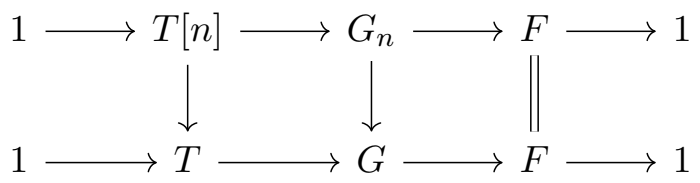

and the associated diagram in Galois cohomology. Let $\bar{\alpha} \in H^{1}(K, F)$ be the image of $\alpha$ under the natural morphism $H^{1}(K, G) \rightarrow H^{1}(K, F)$. Since $T$ is abelian, the conjugation actions of $G$ on $T$ and of $G_{n}$ on $T[n]$ descend to $F$. Twisting the bottom sequence by $\bar{\alpha}$ and setting $U={ }^{\bar{\alpha}} T$, we see that the fiber of $\bar{\alpha}$ equals the image of $H^{1}(K, U)$; see [Ser97, Section I.5.5]. Similarly twisting the top sequence by $\bar{\alpha}$, we see that the fiber of $H^{1}\left(K, G_{n}\right) \rightarrow H^{1}(K, F)$ over $\bar{\alpha}$ equals the image of $H^{1}(K, U[n])$. Here $n$ is a power of $p$. Thus it suffices to prove the following:

Claim. Let $K$ be a $p$-closed field and $U$ be a torus defined over $K$. Then the natural map $H^{1}\left(K, U\left[p^{r}\right]\right) \rightarrow H^{1}(K, U)$ is surjective for $r$ sufficiently large.

To prove the claim, note that since $K$ is $p$-closed, the torus $U$ is split by an extension $L / K$ of degree $n$, where $n$ is a power of $p$. By a restriction-corestriction argument, it follows that $H^{1}(K, U)$ is $n$-torsion. Now consider the short exact sequence

$$
1 \longrightarrow U[n] \longrightarrow U \stackrel{\times n}{\longrightarrow} U \longrightarrow 1 .
$$

The associated exact cohomology sequence

$$
H^{1}(K, U[n]) \longrightarrow H^{1}(K, U) \stackrel{\times n}{\longrightarrow} H^{1}(K, U)
$$

shows that $H^{1}(K, U[n])$ surjects onto $H^{1}(K, U)$. This completes the proof of the claim and thus of the Lemma 5.2. 


\section{Z. Reichstein And F. SCAVia}

\section{The index}

Let $\mu$ be a diagonalizable abelian $p$-group and

$$
1 \longrightarrow \mu \longrightarrow G \longrightarrow \bar{G} \longrightarrow 1
$$

be a central exact sequence of affine algebraic groups defined over $k$. This sequence gives rise to the exact sequence of pointed sets

$$
H^{1}(K, G) \longrightarrow H^{1}(K, \bar{G}) \stackrel{\partial_{K}}{\longrightarrow} H^{2}(K, \mu)
$$

for any field extension $K$ of the base field $k$. Any character $x: \mu \rightarrow \mathbb{G}_{\mathrm{m}}$ induces a homomorphism $x_{*}: H^{2}(K, \mu) \rightarrow H^{2}\left(K, \mathbb{G}_{\mathrm{m}}\right)$. We define $\operatorname{ind}^{x}(G, \mu)$ as the maximal index of $x_{*} \circ \partial_{K}(E) \in$ $H^{2}(K, \mu)$, where the maximum is taken over all field extensions $K / k$ and over all $E \in H^{1}(K, \bar{G})$. This number is finite for every character $x: \mu \rightarrow \mathbb{G}_{\mathrm{m}}$; see [Mer13, Theorem 6.1].

Remark 6.1. Since $\mu$ is a finite $p$-group, the index of $x_{*} \circ \partial_{K}(E)$ does not change when $K$ is replaced by a finite extension $K^{\prime} / K$ whose degree is prime to $p$ and $E$ is replaced by its image under the natural restriction map $H^{1}(K, \bar{G}) \rightarrow H^{1}\left(K^{\prime}, \bar{G}\right)$. Equivalently, we may replace $K$ with its $p$-closure $K^{(p)}$. In other words, the maximal value of $x_{*} \circ \partial_{K}(E)$ will be attained if we only allow $K$ to range over $p$-closed fields extensions of $k$.

Set $\operatorname{ind}(G, \mu):=\min \sum_{i=1}^{r}$ ind $^{x_{i}}(G, \mu)$, where the minimum is taken over all generating sets $x_{1}, \ldots, x_{r}$ of the group $X(\mu)$ of characters of $\mu$.

Now suppose that $G^{0}=T$ is a torus and $G / G^{0}=F$ is a $p$-group, as in (1.1). In this case, there is a particularly convenient choice of $\mu \subset G$. Following [LMMR13b, Section 4], we denote this central subgroup of $G$ by $C(G)$. If $k$ is algebraically closed, $C(G)$ is simply the $p$-torsion subgroup of the center of $G$, that is, $C(G)=Z(G)[p]$. If $k$ is only assumed to be $p$-closed, then we set $C(G)=\operatorname{Split}_{k}(Z(G)[p])$ to be the largest $k$-split subgroup of $Z(G)[p]$ in the sense of [LMMR13a, Section 2].

Proposition 6.2. Let $G$ be as in (1.1). Denote by $\eta(G)$ the smallest dimension of a $p$-faithful $G$-representation.

(a) We have $\operatorname{ind}(G, C(G))=\eta(G)$.

(b) If $r$ is sufficiently large, then $\eta(G)=\eta\left(G_{p^{r}}\right)=\operatorname{ed}\left(G_{p^{r}}\right)=\operatorname{ed}\left(G_{p^{r}} ; p\right)$.

Proof. (a) Let $\operatorname{Rep}^{x}(G)$ be the set of irreducible $G$-representations $\nu: G \rightarrow \operatorname{GL}(V)$ such that $\nu(z)=x(z) \operatorname{Id}_{V}$ for every $z \in \mu(\bar{k})$. By the index formula [Mer13, Theorem 6.1], we have $\operatorname{ind}^{x}(G)=\operatorname{gcd} \operatorname{dim}(\nu)$, where $\nu$ ranges over $\operatorname{Rep}^{x}(G)$ and gcd stands for the greatest common divisor. By [LMMR13b, Proposition 4.2], the $\operatorname{dimension} \operatorname{dim}(\nu)$ is a power of $p$ for every irreducible representation $\nu$ of $G$ defined over $k$. Thus one can replace $\operatorname{gcd} \operatorname{dim}(\nu)$ with $\min \operatorname{dim}(\nu)$ in the index formula. Decomposing an arbitrary representation of $G$ as a direct sum of irreducible subrepresentations, we see that $\operatorname{ind}(G, C(G))$ equals the minimal dimension of a $k$-representation $\nu: G \rightarrow \mathrm{GL}(V)$ such that the restriction $\nu_{\mid C(G)}: C(G) \rightarrow \mathrm{GL}(V)$ is faithful. Finally, by [LMMR13b, Proposition 4.3], the restriction $\nu_{\mid C(G)}$ is faithful if and only if $\nu$ is $p$-faithful.

(b) Since $G_{p^{r}}$ is a (not necessarily constant) finite $p$-group and $k$ is $p$-closed, the identities $\eta\left(G_{p^{r}}\right)=\operatorname{ed}\left(G_{p^{r}}\right)=\operatorname{ed}\left(G_{p^{r}} ; p\right)$ follow from [LMMR13a, Theorem 7.1]. It thus remains to show that

$$
\eta(G)=\eta\left(G_{p^{r}}\right) \quad \text { for } r \gg 0
$$




\section{EXTENSIONS OF FINITE GROUPS BY TORI}

By Lemma 5.1(b), we have $Z(G)[p]=Z\left(G_{p^{r}}\right)[p]$ and thus $C(G)=C\left(G_{p^{r}}\right)$ for $r \gg 0$. In view of part (a), the identity (6.2) is thus equivalent to

$$
\operatorname{ind}(G, C(G))=\operatorname{ind}\left(G_{p^{r}}, C(G)\right) \text { for } r \gg 0 \text {. }
$$

Let $h$ be the natural projection $G \rightarrow \bar{G}=G / C(G)$. Note that the group $\bar{G}$ is of the same type as $G$. That is, the connected component $\bar{G}^{0}$ is the torus $\bar{T}:=h(T)$, and since the homomorphism $F=G / T \rightarrow \bar{G} / \bar{T}$ is surjective, $\bar{F}:=\bar{G} / \bar{G}^{0}$ is a $p$-group. Moreover, if $F^{\prime}$ is a quasi-splitting subgroup for $G$ (as defined at the beginning of Section 5), then $\bar{F}^{\prime}:=h\left(F^{\prime}\right)$ is a quasi-splitting subgroup for $\bar{G}$. We will use this subgroup to define the finite subgroups $\bar{G}_{n}$ of $\bar{G}$ for every integer $n$ in the same way as we defined $G_{n}$ :

$$
\bar{G}_{n} \text { is the subgroup of } \bar{G} \text { generated by } \bar{F}^{\prime} \text { and the torsion subgroup } \bar{T}[n] \text {. }
$$

Now observe that since $C(G)$ is $p$-torsion in $G$, we have $h(T[n]) \subset \bar{T}[n] \subset h(T[p n])$ and thus

$$
h\left(G_{n}\right) \subset \bar{G}_{n} \subset h\left(G_{p n}\right)
$$

for every $n$. We now proceed with the proof of (6.3). Consider the diagram of natural maps

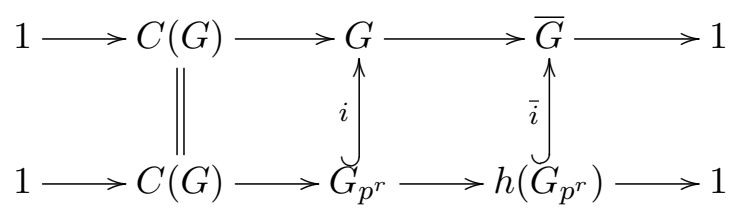

and the induced diagram in Galois cohomology

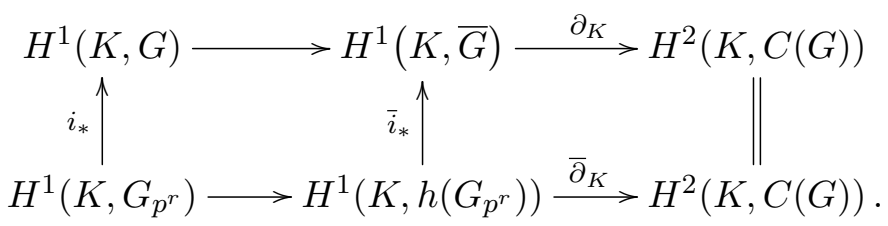

In view of Remark 6.1, for the purpose of computing ind $(G, C(G))$ and $\operatorname{ind}\left(G_{p^{r}}, C(G)\right)$, we may assume that $K$ is a $p$-closed field. We claim that for $r \gg 0$, the vertical map $\bar{i}_{*}: H^{1}\left(K, h\left(G_{p^{r}}\right)\right) \rightarrow$ $H^{1}(K, \bar{G})$ is surjective for every $p$-closed field $K / k$. If we can prove this claim, then for $r \gg 0$, the image of $\bar{\partial}_{K}$ in $H^{2}(K, C(G))$ is the same as the image of $\partial_{K}$. Thus ind ${ }^{x}(G)$ and ind ${ }^{x}\left(G_{p^{r}}\right)$ are the same for every $x \in X(C(G))$, and (6.3) will follow.

To prove the claim, note that $\bar{G}_{p^{r}} \subset h\left(G_{p^{r+1}}\right)$ by (6.4). Consider the composition

$$
H^{1}\left(K, \bar{G}_{p^{r-1}}\right) \longrightarrow H^{1}\left(K, h\left(G_{p^{r}}\right)\right) \stackrel{\bar{i}_{*}}{\longrightarrow} H^{1}(K, \bar{G}) .
$$

By Lemma 5.2, the map $H^{1}\left(K, \bar{G}_{p^{r-1}}\right) \rightarrow H^{1}(K, \bar{G})$ is surjective for $r \gg 0$. Hence, so is $\bar{i}_{*}$. This completes the proof of the claim and thus of (6.3) and of Proposition 6.2.

\section{A resolution theorem for rational maps}

The following lemma is a minor variant of [BRV18, Lemma 2.1]. For the sake of completeness, we supply a self-contained proof.

Lemma 7.1. Let $K \subset L$ be a field extension and $v: L^{\times} \rightarrow \mathbb{Z}$ be a discrete valuation. Assume that $\left.v\right|_{K^{\times}}$is non-trivial, and denote the residue fields of $v$ and $\left.v\right|_{K^{\times}}$by $L_{v}$ and $K_{v}$, respectively. Then $\operatorname{trdeg}_{K} L \geqslant \operatorname{trdeg}_{K_{v}} L_{v}$. 


\section{Z. Reichstein And F. SCAVia}

Proof. Let $\bar{x}_{1}, \ldots, \bar{x}_{m} \in L_{v}$. For every $i$, let $x_{i}$ be a preimage of $\bar{x}_{i}$ in the valuation $\operatorname{ring} \mathcal{O}_{L}$. It suffices to show that if $\bar{x}_{1}, \ldots, \bar{x}_{m}$ are algebraically independent over $K_{v}$, then $x_{1}, \ldots, x_{m}$ are algebraically independent over $K$. To prove this, we argue by contradiction. Suppose that there exists a non-zero polynomial $f \in K\left[t_{1}, \ldots, t_{m}\right]$ such that $f\left(x_{1}, \ldots, x_{m}\right)=0$. Multiplying $f$ by a suitable power of a uniformizing parameter for $\left.v\right|_{K^{\times}}$, we may assume that $f \in \mathcal{O}_{K}\left[x_{1}, \ldots, x_{m}\right]$ and that at least one coefficient of $f$ has valuation equal to 0 . Reducing modulo the maximal ideal of the valuation ring $\mathcal{O}_{K}$, we see that $\bar{x}_{1}, \ldots, \bar{x}_{m}$ are algebraically dependent over $K_{v}$, which leads to a contradiction.

Recall that if $X_{1}$ is normal and $X_{2}$ is complete, any rational map $f: X_{1} \rightarrow X_{2}$ is regular in codimension 1. It follows that if $D \subset X_{1}$ is a prime divisor of $X_{1}$, the closure of the image $\overline{f(D)} \subset X_{2}$ is well defined.

Theorem 7.2. Let $G$ be a smooth linear algebraic group over $k$ and $f: X \rightarrow Y$ be a dominant rational map of $G$-varieties. Assume that $X$ is normal, $Y$ is normal and complete, $D \subset X$ is a prime divisor, and $\overline{f(D)} \neq Y$. Then there exist a commutative diagram of $G$-equivariant dominant rational maps

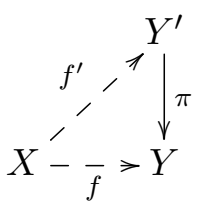

and a divisor $E \subset Y^{\prime}$ such that $Y^{\prime}$ is complete, $\pi: Y^{\prime} \rightarrow Y$ is a birational morphism, and $\overline{f^{\prime}(D)}=E$.

Proof. Let $v: k(X)^{\times} \rightarrow \mathbb{Z}$ be the valuation given by the order of vanishing or pole along $D$. Since $X$ is normal and $Y$ is complete, $f$ restricts to a rational map $D \rightarrow Y$. Denote the Zariski closure of the image of this map by $C$, and set

$$
w: k(Y)^{\times} \stackrel{f^{*}}{\longrightarrow} k(X)^{\times} \stackrel{v}{\longrightarrow} \mathbb{Z} .
$$

We claim that $w$ is non-zero; that is, $w$ is a discrete valuation on $k(Y)$. Indeed, choose $\varphi \in k(Y)^{\times}$ so that $\varphi$ is regular in an open neighborhood $U$ of the generic point of $C$ and $\left.\varphi\right|_{U \cap C}=0$. It follows that $\varphi \circ f$ is zero on $D$, hence $w(f)=v(\varphi \circ f)>0$. This proves the claim.

Since $D$ maps dominantly onto $C$, we have an inclusion of local rings $f^{*}: \mathcal{O}_{Y, C} \hookrightarrow \mathcal{O}_{X, D}$. It follows that if $\varphi \in \mathcal{O}_{Y, C}$, then $w(\varphi)=v(\varphi \circ f) \geqslant 0$; that is, $\mathcal{O}_{Y, C}$ is contained in the valuation ring of $w$. In other words, $C$ is the center of $w$.

Denote by $k(Y)_{w}$ the residue field of $w$. By Lemma 7.1, we have

$$
\operatorname{trdeg}_{k} k(X)-\operatorname{trdeg}_{k} k(Y) \geqslant \operatorname{trdeg}_{k} k(D)-\operatorname{trdeg}_{k} k(Y)_{w} .
$$

Since $\operatorname{trdeg}_{k} k(D)=\operatorname{trdeg}_{k} k(X)-1$, this can be rewritten as

$$
\operatorname{trdeg}_{k} k(Y)_{w} \geqslant \operatorname{trdeg}_{k} k(Y)-1 .
$$

On the other hand, we have $\operatorname{trdeg}_{k} k(Y)_{w} \leqslant \operatorname{trdeg}_{k} k(Y)-1$ by the Zariski-Abhyankar inequality [Bou89, Section VI.10.3, Corollary 1], hence

$$
\operatorname{trdeg}_{k} k(Y)_{w}=\operatorname{trdeg}_{k} k(Y)-1 .
$$

By [Art86, Theorem 5.2], there exists a sequence of proper birational morphisms

$$
Y^{\prime}=Y_{n} \rightarrow Y_{n-1} \rightarrow \cdots \rightarrow Y_{1} \rightarrow Y_{0}=Y
$$




\section{EXTENSIONS OF FINITE GROUPS BY TORI}

such that each $Y_{i+1} \rightarrow Y_{i}$ is a blow-up at the center of $w$ on $Y_{i}$, the center $E^{\prime}$ of $w$ on $Y^{\prime}$ is a prime divisor, and $Y^{\prime}$ is normal at the generic point of $E^{\prime}$. Since $C$ is $G$-invariant, by the universal property of the blow-up, the $G$-action on $Y$ lifts to every $Y_{i}$, and the maps $Y_{i+1} \rightarrow Y_{i}$ are $G$-equivariant.

We let $\pi: Y^{\prime} \rightarrow Y$ be the composition of the maps $Y_{i+1} \rightarrow Y_{i}$ and $f^{\prime}: X \rightarrow Y^{\prime}$ be the composition of $f$ with the birational inverse of $\pi$. By construction, $f^{\prime}$ is $G$-equivariant. It suffices to show that $\overline{f^{\prime}(D)}=E$. Since the center of $w$ is the divisor $E \subset Y^{\prime}$, the valuation $w$ is given by the order of vanishing or pole along $E$. If we identify $k(Y)$ with $k\left(Y^{\prime}\right)$ via $\pi$, we also have $w=\left(f^{\prime}\right)^{*} v$. It follows that $\varphi \in k\left(Y^{\prime}\right)^{\times}$is regular and vanishes at the generic point of $E$ if and only if $w(\varphi)>0$ if and only if $v\left(\varphi \circ f^{\prime}\right)>0$ if and only if $\varphi$ vanishes at the generic point of $f^{\prime}(D)$. We conclude that $\overline{f^{\prime}(D)}=E$, as desired.

Since $G$ is smooth, the $G$-action on $Y^{\prime}$ lifts to the normalization $\left(Y^{\prime}\right)^{\text {norm }}$, so that the normalization map $\left(Y^{\prime}\right)^{\text {norm }} \rightarrow Y^{\prime}$ is $G$-equivariant. After replacing $Y^{\prime}$ with $\left(Y^{\prime}\right)^{\text {norm }}$ and $E^{\prime}$ with its preimage in $\left(Y^{\prime}\right)^{\text {norm }}$, we may assume that $Y^{\prime}$ is normal everywhere (and not just at the generic point of $\left.E^{\prime}\right)$.

\section{Proof of Theorem 1.2}

Let $G$ be an algebraic group as in (1.1). Let $\nu: G \rightarrow \operatorname{GL}(V)$ be a $p$-faithful representation of $G$ of minimal dimension $\eta(G)$. By Lemma 2.1, there exists a stabilizer in general position $S_{V}$ for the $G_{\bar{k}^{-}}$action on $V_{\bar{k}}$. Since $V(k)$ is dense in $V$, we may assume without loss of generality that $S_{V}$ is the stabilizer of a $k$-point of $V$. In particular, we may assume that $S_{V}$ is a closed subgroup of $G$ defined over $k$. Since $T$ acts $p$-faithfully on $V$, we have $S_{V} \cap T=\{1\}$.

Reduction 8.1. To prove Theorem 1.2, it suffices to construct a $G$-representation $V^{\prime}$ such that $\operatorname{dim}(\tilde{V})=\operatorname{rank}_{p}\left(S_{V}\right), W:=V \oplus \tilde{V}$ is $p$-generically free, and

$$
\operatorname{ed}(W ; p)=\operatorname{dim}(W)-\operatorname{dim}(G) .
$$

Here when we write $\operatorname{ed}(W ; p)$, we are viewing $W$ as a generically free $G / \operatorname{Ker}(\varphi)$-variety, where $\varphi: G \rightarrow \operatorname{GL}(W)$ denotes the representation of $G$ on $W$. The kernel $\operatorname{Ker}(\varphi)$ of this representation is a finite normal subgroup of $G$ of order prime to $p$.

Proof. Suppose that we manage to construct $\tilde{V}$ so that (8.1) holds. Then

$$
\operatorname{ed}(W ; p) \stackrel{(\mathrm{i})}{=} \operatorname{ed}(G / \operatorname{Ker}(\varphi) ; p) \stackrel{(\mathrm{ii})}{=} \operatorname{ed}(G ; p) \stackrel{(\mathrm{iii})}{\leqslant} \rho(G)-\operatorname{dim}(G) \stackrel{(\mathrm{iv})}{\leqslant} \operatorname{dim}(W)-\operatorname{dim}(G),
$$

where

(i) follows from the fact that $W$ is a versal $G / \operatorname{Ker}(\varphi)$-variety; see, for example, [Mer13, Propositions 3.10 and 3.11];

(ii) follows by [LMMR13b, Proposition 2.4];

(iii) is the right-hand side of (1.2); and

(iv) is immediate from the definition of $\rho(G)$.

If we know that (8.1) holds, then the inequalities (iii) and (iv) are, in fact, equalities. Equality in (iii) yields Theorem 1.2(a). On the other hand, since

$$
\operatorname{dim}(W)=\operatorname{dim}(V)+\operatorname{dim}(\tilde{V})=\eta(G)+\operatorname{rank}_{p}\left(S_{V}\right),
$$

equality in (iv) tells us that $\eta(G)+\operatorname{rank}_{p}\left(S_{V}\right)=\rho(G)$, thus proving Theorem 1.2(b). 


\section{Z. Reichstein AND F. Scavia}

We now proceed with the construction of $W$. From now on, we replace $G$ with $\bar{G}=G / \operatorname{Ker}(\nu)$. All other $G$-actions we will construct (including the linear $G$-action on $W$ ) will factor through $\bar{G}$. In the end, we will show that $\operatorname{ed}(W ; p)=\operatorname{ed}(\bar{G} ; p)$; once again, this is enough because $\operatorname{ed}(G ; p)=\eta(G)=\eta(\bar{G})=\operatorname{ed}(\bar{G} ; p)$ by [LMMR13b, Proposition 2.4]. In other words, from now on we may (and will) assume that the $G$-action on $V$ is faithful.

Recall that $S_{V}$ denotes the stabilizer in general position for the $G$-action on $V$ and that we have chosen $S_{V}$ (which is a priori a closed subgroup of $G_{\bar{k}}$ defined up to conjugacy) so that it is defined over $k$. Since $T$ is a torus and $T$ acts faithfully on $V$, this action is automatically generically free. That is, $S_{V} \cap T=1$ or, equivalently, the natural projection $\left.\pi\right|_{S_{V}}: S_{V} \rightarrow F$ is injective. In particular, $\pi\left(S_{V}\right)$ is diagonalizable. By our assumption, $F$ is isomorphic to the product $\mu_{p^{i_{1}}} \times \cdots \times \mu_{p^{i_{R}}}$ for some integers $R \geqslant 0$ and $i_{1}, \ldots, i_{R} \geqslant 1$. Moreover, this isomorphism can be chosen so that

$$
\pi\left(S_{V}\right)=\mu_{p^{j_{1}}} \times \cdots \times \mu_{p^{j_{r}}}
$$

for some $0 \leqslant r \leqslant R$ and some integers $j_{m}$ with $1 \leqslant j_{m} \leqslant i_{m}$ for every $m=1, \ldots, r$. Let $\chi_{m}$ be the composition of $\pi: G \rightarrow F$ with the projection map $F \rightarrow \mu_{p^{i m}}$ to the $m$ th component and $V_{m}$ be a 1-dimensional vector space on which $G$ acts by $\chi_{m}$. Set $W_{d}=V$ and $W_{d+m}=V \oplus V_{1} \oplus \cdots \oplus V_{m}$ for $m=1, \ldots, r$. A stabilizer in general position for the $G$-action on $W_{d+m}$ is

$$
S_{W_{d+m}}=S_{V} \cap \operatorname{Ker}\left(\chi_{1}\right) \cap \cdots \cap \operatorname{Ker}\left(\chi_{m}\right) ;
$$

equivalently,

$$
S_{W_{d+m}} \simeq \pi\left(S_{W_{d+m}}\right)=\{1\} \times \cdots \times\{1\} \times \mu_{p^{j_{m+1}}} \times \cdots \times \mu_{p^{j}}
$$

for any $0 \leqslant m \leqslant r$. In particular, $S_{W_{d+r}}=\{1\}$; in other words, the $G$-action on $W_{d+r}$ is generically free. We now set

$$
W=W_{d+r}=V \oplus V_{1} \oplus \cdots \oplus V_{r} .
$$

Having defined $W$, we now proceed with the proof of (8.1). In view of Lemma 4.1(b), it suffices to establish the following.

Proposition 8.2. Let $W$ be as above. Consider a dominant $G$-equivariant correspondence

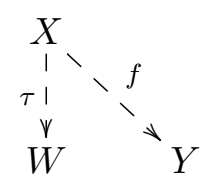

of degree prime to $p$, where $Y$ is a p-generically free projective $G$-variety. Then $\operatorname{dim}(Y)=$ $\operatorname{dim}(W)=d+r$.

We now proceed with the proof of the proposition. By Lemma 3.1 (with $Z=W_{d+r-1}$ ), there exists a commutative diagram of $G$-equivariant maps

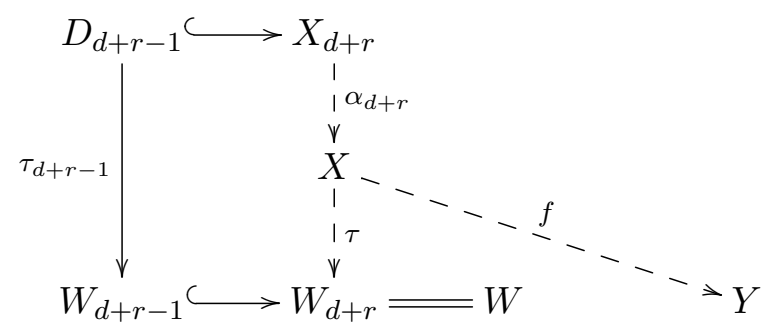




\section{EXTENSIONS OF FINITE GROUPS BY TORI}

such that $X_{d+r}$ is normal, $\alpha_{d+r}$ is a birational isomorphism, $D_{d+r-1}$ is an irreducible divisor in $X_{d+r}$, and $\tau_{d+r-1}$ is a cover of $W_{d+r-1}$ of degree prime to $p$. Let $S_{D_{d+r-1}} \subset G$ be a stabilizer in general position for the $G$-action on $D_{d+r-1}$; it exists by Lemma 2.1. In view of (8.2), Lemma 3.2 tells us that

$$
\operatorname{rank}_{p}\left(S_{D_{d+r-1}}\right)=1 .
$$

On the other hand, by our assumption, the $G$-action on $Y$ is $p$-generically free. Thus the restriction ${ }^{1}$ of the dominant rational map $f \circ \alpha_{d+r}: X_{d+r} \rightarrow Y$ to $D_{d+r-1}$ cannot be dominant, and Theorem 7.2 applies: there exists a commutative diagram

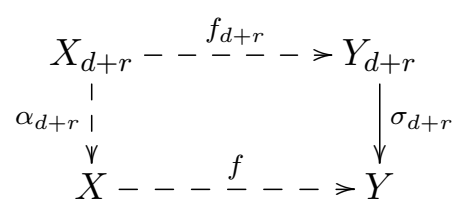

of dominant $G$-equivariant rational maps, where $\sigma_{d+r}$ is a birational morphism, $Y_{d+r}$ is normal and complete, and $f_{d+r}$ restricts to a dominant $G$-equivariant rational map $D_{d+r-1}-\rightarrow E_{d+r-1}$ for some $G$-invariant irreducible divisor $E_{d+r-1}$ of $Y_{d+r}$. We will denote this dominant rational map by $f_{d+r-1}: D_{d+r-1} \rightarrow E_{d+r-1}$. We now iterate this construction with $f_{d+r}$ replaced by $f_{d+r-1}$.

By Lemma 3.1, there exists a commutative diagram of $G$-equivariant maps

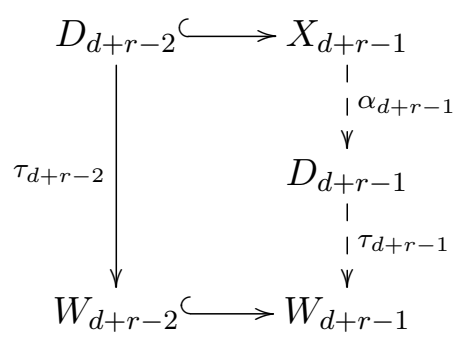

such that $X_{d+r-1}$ is normal, $\alpha_{d+r-1}$ is a birational isomorphism, $D_{d+r-2}$ is an irreducible divisor in $X_{d+r-1}$, and $\tau_{d+r-2}$ is a cover of $W_{d+r-2}$ of degree prime to $p$.

Denote a stabilizer in general position for the $G$-action on $E_{d+r-1}$ by $S_{E_{d+r-1}}$. Recall that the $G$-action on $Y$ (and thus $Y_{d+r}$ ) is $p$-generically free. Since $E_{d+r-1}$ is a $G$-invariant hypersurface in $Y_{d+r}$, Lemma 2.3(a) tells us that $\operatorname{rank}_{p}\left(S_{E_{d+r-1}}\right) \leqslant 1$. On the other hand, since $X_{d+r-1}$ maps dominantly to $E_{d+r-1}$, the group $S_{E_{d+r-1}}$ contains (a conjugate of) $S_{X_{d+r-1}}$ and thus $\operatorname{rank}_{p}\left(S_{E_{d+r-1}}\right) \geqslant \operatorname{rank}_{p}\left(S_{X_{d+r-1}}\right)$, where $\operatorname{rank}_{p}\left(S_{X_{d+r-1}}\right)=1$ by (8.3). We conclude that $\operatorname{rank}_{p}\left(S_{E_{d+r-1}}\right)=1$. Now observe that $\operatorname{since} \operatorname{rank}_{p}\left(S_{E_{d+r-1}}\right)=1$ and $\operatorname{rank}_{p}\left(S_{X_{d+r-2}}\right)=2$ (see (8.2)), the image of $X_{d+r-2}$ under $f_{d+r-1}$ cannot be Zariski dense in $E_{d+r-1}$. Consequently, Theorem 7.2 can be applied to $f_{d+r-1}: X_{d+r-1} \rightarrow E_{d+r-1}$. It yields a birational morphism $\sigma_{d+r-1}: Y_{d+r-1} \rightarrow E_{d+r-1}$ such that $Y_{d+r-1}$ is normal and complete, and the composition $\sigma_{d+r-1}^{-1} \circ f_{d+r-1}$ restricts to a dominant $G$-equivariant rational map $f_{d+r-2}: D_{d+r-2}-\rightarrow E_{d+r-2}$ for some $G$-invariant prime divisor $E_{d+r-2}$ of $Y_{d+r-1}$. Proceeding recursively, we obtain a commutative diagram of $G$-equivariant maps

\footnotetext{
${ }^{1}$ The restriction of $f \circ \alpha_{d+r}$ to $D_{d+r-1}$ is well defined because $X_{d+r}$ is normal and $Y$ is complete.
} 


\section{Z. Reichstein And F. Scavia}

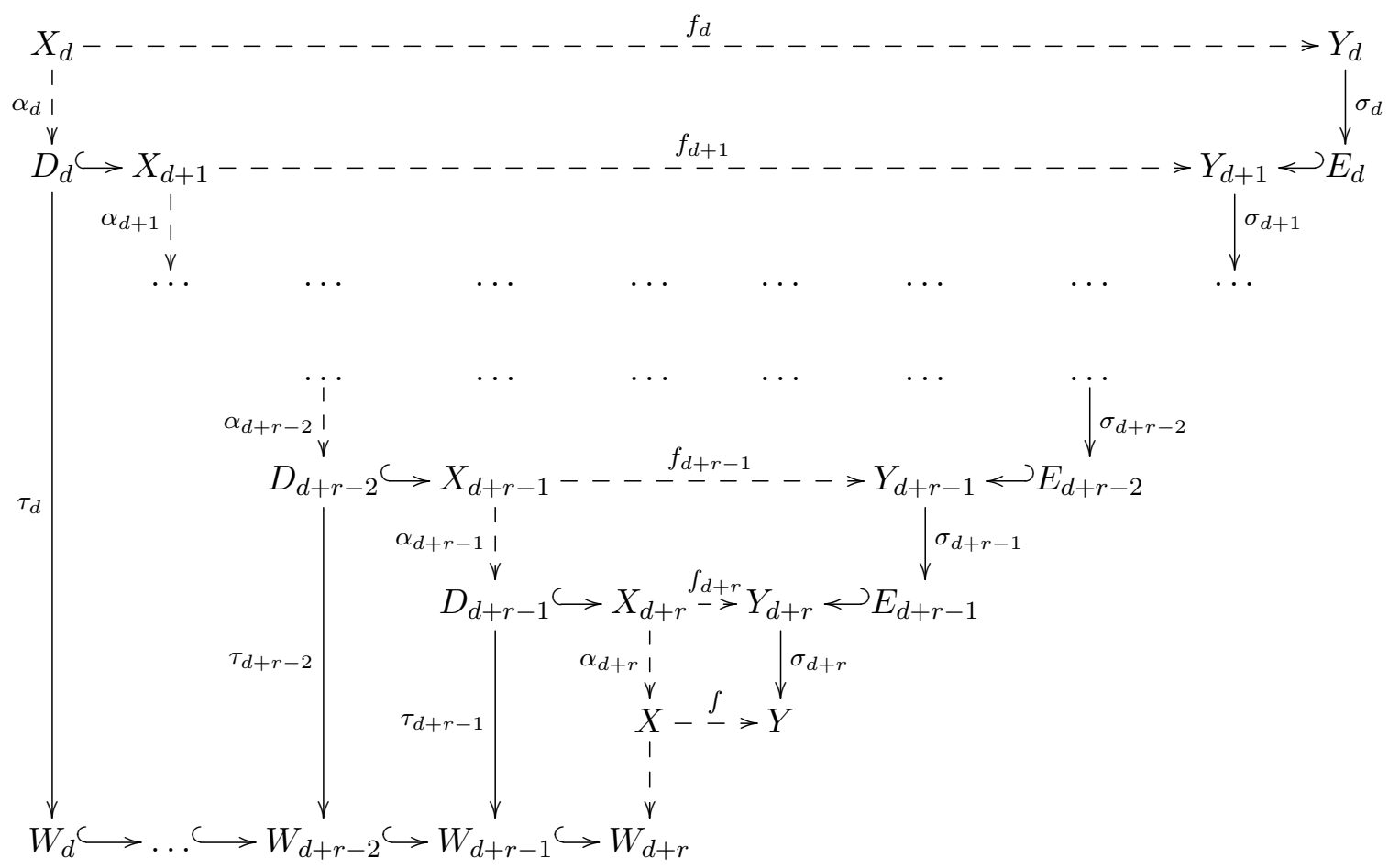

such that for every $m$,

(i) $D_{d+m}$ is an irreducible divisor in $X_{d+m+1}$ and $E_{d+m}$ is an irreducible divisor in $Y_{d+m+1}$;

(ii) the vertical maps $\alpha_{d+m}: X_{d+m} \rightarrow D_{d+m}$ and $\sigma_{d+m}: Y_{d+m} \rightarrow E_{d+m}$ are birational isomorphisms;

(iii) $X_{d+m}$ and $Y_{d+m}$ are normal, and $Y_{d+m}$ is complete;

(iv) $\operatorname{rank}_{p}\left(S_{X_{d+m}}\right)=\operatorname{rank}_{p}\left(S_{Y_{d+m}}\right)=r-m$;

(v) the vertical morphism $\tau_{d+m}: D_{d+m} \rightarrow W_{d+m}$ is a cover of degree prime to $p$.

Note that the subscripts are chosen so that $\operatorname{dim}\left(X_{d+m}\right)=\operatorname{dim}\left(W_{d+m}\right)=d+m$ for each $m=$ $0, \ldots, r$. We will eventually show that $\operatorname{dim}\left(Y_{d+m}\right)=d+m$ for each $m$ as well, but we do not know what $\operatorname{dim}\left(Y_{d+m}\right)$ is at this point.

Lemma 8.3. The $G$-action on $Y_{d+m}$ (or, equivalently, on $\left.E_{d+m}\right)$ is $p$-faithful for every $m=0, \ldots, r$.

Assume, for a moment, that this lemma is established. By our construction, $f_{d}$ may be viewed as a dominant $G$-equivariant correspondence $W_{d} \rightsquigarrow Y_{d}$ of degree prime to $p$. Now recall that $W_{d}=V$ is a $p$-faithful representation of $G$ of minimal possible dimension $\eta(G)$. By Lemma 8.3, the $G$-action of $Y_{d}$ is $p$-faithful. Restricting to the $p$-subgroup $G_{n} \subset G$, where $n$ is a power of $p$, we obtain a dominant $G_{n}$-equivariant correspondence $f_{d}: V \rightsquigarrow Y_{d}$ of degree prime to $p$, where the $G_{n}$-action on $Y$ is faithful. Thus $\operatorname{dim}\left(Y_{d}\right) \geqslant \operatorname{ed}\left(G_{n} ; p\right)$. When $n$ is a sufficiently high power of $p$, Proposition 6.2 tells us that

$$
\operatorname{ed}\left(G_{n} ; p\right)=\eta\left(G_{n}\right)=\eta(G)=\operatorname{dim}(V)=d .
$$

By conditions (i) and (ii) above, $\operatorname{dim}\left(Y_{d+m+1}\right)=\operatorname{dim}\left(E_{d+m}\right)+1=\operatorname{dim}\left(Y_{d+m}\right)+1$ for each $m=0,1, \ldots, r$. Thus $\operatorname{dim}(Y)=\operatorname{dim}\left(Y_{d+r}\right)=\operatorname{dim}\left(Y_{d}\right)+r=\operatorname{dim}(V)+r=d+r=\operatorname{dim}(W)$, as desired. This will complete the proof of Proposition 8.2 and thus of Theorem 1.2. 


\section{EXTENSIONS OF FINITE GROUPS BY TORI}

Proof of Lemma 8.3. For the purpose of this proof, we may replace $k$ with its algebraic closure $\bar{k}$ and thus assume that $k$ is algebraically closed. We argue by reverse induction on $m$. For the base case, where $m=r$, note that by our assumption, the $G$-action on $Y$ is $p$-generically free and hence $p$-faithful. Since $Y_{d+r}$ is birationally isomorphic to $Y$, the same is true of the $G$-action on $Y_{d+r}$.

For the induction step, assume that the $G$-action on $Y_{d+m+1}$ is $p$-faithful for some $m$ with $0 \leqslant m \leqslant r-1$. Our goal is to show that the $G$-action on $Y_{d+m}$ is also $p$-faithful. Let $N$ be the kernel of the $G$-action on $Y_{d+m}$. Recall that by Lemma 2.3(b), there is a homomorphism

$$
\alpha: N \rightarrow \mathbb{G}_{\mathrm{m}}
$$

where $\operatorname{Ker}(\alpha)$ has no elements of order $p$. Since $\operatorname{Ker}(\alpha)$ is a subgroup of $G$ and we are assuming that $G^{0}=T$ is a torus and $G / G^{0}=F$ is a finite $p$-group, we conclude that

$$
\operatorname{Ker}(\alpha) \text { is a finite subgroup of } T \text { of order prime to } p \text {. }
$$

It remains to show that $\alpha(N)$ is a finite group of order prime to $p$. Assume the contrary: $\alpha(N)$ contains $\mu_{p} \subset \mathbb{G}_{\mathrm{m}}$.

Claim. There exists a subgroup $\mu_{p} \simeq N_{0} \subset N$ such that $N_{0}$ is central in $G$.

First we observe that in order to prove the claim, it suffices to show that there exists a subgroup $\mu_{p} \simeq N_{0} \subset N$ such that $N_{0}$ is normal in $G$. Indeed, since $G^{0}=T$ is a torus and $G / G^{0}=F$ is a $p$-group, if $N_{0} \simeq \mu_{p}$ is normal in $G$, then the conjugation map $G \rightarrow \operatorname{Aut}\left(\mu_{p}\right) \simeq \mathbb{Z} /(p-1) \mathbb{Z}$ is trivial, so $N_{0}$ is automatically central. Now consider two cases.

Case 1: $G^{0}=T$ does not act $p$-faithfully on $Y_{d+m}$. Then $\mu_{p} \subset N \cap T \triangleleft G$. In view of (8.4) and (8.5), the intersection $N \cap T$ contains exactly one copy of $\mu_{p}$. This implies that $\mu_{p}$ is characteristic in $N \cap T$ and, hence, normal in $G$, as desired.

Case 2: The intersection $N \cap T$ does not contain $\mu_{p}$; that is, $N \cap T$ is a finite group of order prime to $p$. Examining the exact sequence

$$
1 \rightarrow N \cap T \rightarrow N \rightarrow F=G / T,
$$

we see that $N$ is a finite group of order $p m$, where $m$ is prime to $p$. Let $\operatorname{Syl}_{p}(N)$ be the set of $\operatorname{Syllow}$ $p$-subgroups of $N$. By Sylow's theorem, we have ${ }^{2}\left|\operatorname{Syl}_{p}(N)\right| \equiv 1(\bmod p)$. The group $G$ acts on $\operatorname{Syl}_{p}(N)$ by conjugation. Clearly $T$ acts trivially, and the $p$-group $F=G / T$ fixes a subgroup $N_{0} \in \operatorname{Syl}_{p}$. In other words, $N_{0} \simeq \mu_{p}$ is normal in $G$. This proves the claim.

We are now ready to finish the proof of Lemma 8.3. Let $S_{Y_{d+m}} \subset G$ be a stabilizer in general position for the $G$-action on $Y_{d+m}, N$ be the kernel of this action, and $N_{0}$ be the central subgroup of $N$ isomorphic to $\mu_{p}$, as in the claim. Clearly $N_{0} \subset N \subset S_{Y_{d+m}}$. Since $f_{d+m}: X_{d+m}-\rightarrow Y_{d+m}$ is a dominant $G$-equivariant rational map, $S_{Y_{d+m}}$ contains (a conjugate of) $S_{X_{d+m}}$. By condition (iv),

$$
\operatorname{rank}_{p}\left(S_{Y_{d+m}}\right)=r-m=\operatorname{rank}_{p}\left(S_{X_{d+m}}\right) .
$$

In particular, $S_{X_{d+m}}$ contains a subgroup $A$ isomorphic to $\mu_{p}^{r-m}$. Since $N_{0} \simeq \mu_{p}$ is central in $G$, it has to be contained in $A$; otherwise, $S_{Y_{d+m}}$ would contain a subgroup isomorphic to $A \times \mu_{p}=\left(\mu_{p}\right)^{r-m+1}$, contradicting (8.6). Thus $\mu_{p} \simeq N_{0} \subset S_{X_{d+m}}$. Moreover, since $N_{0}$ is central in $G$, it is contained in every conjugate of $S_{X_{d+m}}$. This implies that $N_{0}$ stabilizes every point

\footnotetext{
${ }^{2}$ Recall that we are assuming that $k$ is an algebraically closed field of characteristic different from $p$. If $\operatorname{char}(k)$ does not divide $|N|$, then $\operatorname{Syl}_{p}(N)$ is the set of Sylow subgroups of the finite group $N(k)$. If $\operatorname{char}(k)$ divides $|N|$, then elements of $\operatorname{Syl}_{p}(N)$ can be identified with Sylow $p$-subgroups of the finite group $N_{\text {red }}(k)$.
} 


\section{Z. Reichstein And F. SCAVia}

of $X_{d+m}$. In other words, $N_{0}$ acts trivially on $X_{d+m}$. Tracing to the above diagram, we see that $N_{0}$ acts trivially on $D_{d+m-1}$, hence on $X_{d+m-1}$, hence on $D_{d+m-2}$, etc. Finally, we conclude that $N_{0}$ acts trivially on $X_{d}$ and hence on $\tau_{d}\left(X_{d}\right)=W_{d}=V$, contradicting our assumption that $G$ acts $p$-faithfully on $W_{d}=V$.

This contradiction shows that our assumption that $\alpha(N)$ contains $\mu_{p}$ was false. Returning to (8.4) and (8.5), we deduce that the kernel $N$ of the $G$-action on $Y_{d+m}$ is a finite group of order prime to $p$. In other words, the $G$-action on $Y_{d+m}$ is $p$-faithful. This completes the proof of Lemma 8.3 and thus of Proposition 8.2 and Theorem 1.2.

Remark 8.4. Our proof of Theorem 1.2 goes through even if $F$ is not abelian, provided that the stabilizer in general position $S_{V}$ projects isomorphically to $F /[F, F]$. (If $F$ is abelian, this is always the case.)

Remark 8.5. Theorem 1.2 implies that if $V$ and $V^{\prime}$ are $p$-faithful representations of $G$ of minimal dimension $\eta(G)$, then the stabilizers in general position, $S_{V}$ and $S_{V^{\prime}}$, have the same $p$-rank:

$$
\operatorname{rank}_{p}\left(S_{V}\right)=\operatorname{rank}_{p}\left(S_{V^{\prime}}\right)=\rho(G)-\eta(G)=\operatorname{ed}(G ; p)+\operatorname{dim}(G)-\eta(G) .
$$

In our proof of Theorem 1.2, this number is denoted by $r$.

\section{Normalizers of maximal tori in split simple groups}

In this section, $\Gamma$ will denote a split simple algebraic group over $k, T$ will denote a $k$-split maximal torus of $\Gamma, N$ will denote the normalizer of $T$ in $\Gamma$, and $W=N / T$ will denote the Weyl group. These groups fit into an exact sequence

$$
1 \longrightarrow T \longrightarrow N \stackrel{\pi}{\longrightarrow} W \longrightarrow 1 \text {. }
$$

A. Meyer and the first author [MR09] have computed ed $(N ; p)$ in the case where $\Gamma=\mathrm{PGL}_{n}$ for every prime number $p$. M. MacDonald [Mac11] subsequently found the exact value of $\operatorname{ed}(N ; p)$ for most other split simple groups $\Gamma$. One reason this is of interest is that

$$
\operatorname{ed}(N ; p) \geqslant \operatorname{ed}(\Gamma ; p) ;
$$

see, for example, [Mer13, Section 10a]. Let $W_{p}$ denote a Sylow $p$-subgroup of $W$ and $N_{p}$ denote the preimage of $W_{p}$ in $N$. Then

$$
\operatorname{ed}(N ; p)=\operatorname{ed}\left(N_{p} ; p\right)
$$

see [MR09, Lemma 4.1]. The exact sequence

$$
1 \longrightarrow T \longrightarrow N_{p} \stackrel{\pi}{\longrightarrow} W_{p} \longrightarrow 1
$$

is of the form of (1.1), and thus the inequalities (1.2) apply to $N_{p}$. MacDonald computed the exact value of $\operatorname{ed}(N ; p)=\operatorname{ed}\left(N_{p} ; p\right)$ for most split simple linear algebraic groups $\Gamma$ by showing that the left-hand side and right-hand side of the inequalities (1.2) for $N_{p}$ coincide. There are two families of groups $\Gamma$ where the exact value of $\operatorname{ed}(N ; p)$ remained inaccessible by this method, $\Gamma=\mathrm{SL}_{n}$ and $\Gamma=\mathrm{SO}_{4 n} \cdot{ }^{3}$ As an application of Theorem 1.2, we will now compute ed $(N ; p)$ in these two remaining cases. Our main results are Theorems 9.1 and 9.2 below.

\footnotetext{
${ }^{3}$ The omission of $\mathrm{SL}_{n}$ from [Mac11, Remark 5.11] is an oversight; we are grateful to Mark MacDonald for clarifying this point for us.
} 


\section{EXTENSIONS OF FINITE GROUPS BY TORI}

Theorem 9.1. Let $n \geqslant 1$ be an integer, and let $N$ be the normalizer of a $k$-split maximal torus $T$ in $\mathrm{SL}_{n}$. Then

(a) $\operatorname{ed}(N ; p)=n / p+1$ if $p \geqslant 3$ and $n$ is divisible by $p$,

(b) $\operatorname{ed}(N ; p)=n / 2+1$ if $p=2$ and $n$ is divisible by 4 ,

(c) $\operatorname{ed}(N ; p)=\lfloor n / p\rfloor$ if $p \geqslant 3$ and $n$ is not divisible by $p$,

(d) $\operatorname{ed}(N ; p)=\lfloor n / 2\rfloor$ if $p=2$ and $n$ is not divisible by 4 .

TheOREM 9.2. Let $k$ be a field of characteristic different from 2 and $n \geqslant 1$ be an integer. Let $N$ be the normalizer of a $k$-split maximal torus of $\mathrm{SO}_{4 n}$. Then $\operatorname{ed}(N ; 2)=4 n$.

Our proofs of these theorems will rely on the following simple lemma, which is implicit in [MR09] and [Mac11]. Let $F$ be a finite discrete $p$-group, and let $M$ be an $F$-lattice. The symmetric $p$-rank of $M$ is the minimal cardinality $d$ of a finite $H$-invariant $p$-spanning subset $\left\{x_{1}, \ldots, x_{d}\right\} \subset M$. Here " $p$-spanning" means that the index of the $\mathbb{Z}$-module spanned by $x_{1}, \ldots, x_{d}$ in $M$ is finite and prime to $p$. Following MacDonald, we will denote the symmetric $p$-rank of $M$ by $\operatorname{SymRank}(M ; p)$.

Lemma 9.3. Consider an exact sequence $1 \rightarrow T \rightarrow G \rightarrow F \rightarrow 1$ of algebraic groups over $k$, as in (1.1). Assume further that $T$ is a split torus and $F$ is a constant finite p-group. Denote the character lattice of $T$ by $X(T)$, we will view it as an $F$-lattice. Then $\eta(G) \geqslant \operatorname{SymRank}(X(T) ; p)$.

Here $\eta(G)$ denotes the minimal dimension of a $p$-faithful representation of $G$, as defined in the introduction, and $X(T)$ is viewed as an $F$-lattice. If we further assume that the sequence (1.1) in Lemma 9.3 is split, then, in fact, $\eta(G)=\operatorname{SymRank}(X(T) ; p)$. We shall not need this equality, so we leave its proof as an exercise for the reader.

Proof of Lemma 9.3. Let $V$ be a $p$-faithful representation of $G$ of minimal dimension $r=\eta(G)$. As a $T$-representation, $V$ decomposes as the direct sum of characters $\chi_{1}, \ldots, \chi_{r}$. A simple calculation shows that the $F$-action permutes the $\chi_{i}$. Let $S \subset \mathrm{GL}(V)$ be the diagonal torus generated by the images of the $\chi_{i}$. By construction, the kernel of the $F$-equivariant homomorphism

$$
\left(\chi_{1}, \ldots, \chi_{r}\right): T \rightarrow S
$$

is finite and of order prime to $p$. Passing to character lattices, we obtain an $F$-equivariant homomorphism $X(S) \rightarrow X(T)$ whose cokernel is finite and of order prime to $p$. In other words, the images of the $\chi_{i}$ in $X(T)$ form a $p$-spanning subset of $X(T)$ of size $r$. We conclude that $\operatorname{SymRank}(X(T) ; p) \leqslant r=\eta(G)$, as claimed.

For the proof of Theorem 9.1 we will also need the following lemma. Let $\Gamma=\mathrm{SL}_{n}, T$ be the diagonal maximal torus, $N$ be the normalizer of $T$ in $\mathrm{SL}_{n}, H$ be a subgroup of the Weyl group $W=N / T \simeq \mathrm{S}_{n}$, and $N^{\prime}$ be the preimage of $H$ in $N$. Restricting (9.1) to $N^{\prime}$, we obtain an exact sequence

$$
1 \longrightarrow T \longrightarrow N^{\prime} \stackrel{\pi}{\longrightarrow} H \longrightarrow 1 \text {. }
$$

Lemma 9.4. Let $V_{n}$ be the natural n-dimensional representation of $\mathrm{SL}_{n}$ and $S$ be the stabilizer in general position for the restriction of this representation to $N^{\prime}$. Then (a) $S \cap T=1$ and (b) $\pi(S)=H \cap \mathrm{A}_{n}$.

Here, as usual, $\mathrm{A}_{n}$ denotes the alternating subgroup of $\mathrm{S}_{n}$. 


\section{Z. Reichstein And F. SCAVia}

Proof. Part (a) follows from the fact that the $T$-action on $V_{n}$ is generically free. To prove part (b), note that $\pi(S)$ is the kernel of the action of $H$ on $V_{n} / T$, where $V_{n} / T$ is the rational quotient of $V_{n}$ by the action of $T$; see, for example, the proof of [LMMR13b, Proposition 7.2]. Consider the dense open subset $\mathbb{G}_{\mathrm{m}}^{n} \subset V_{n}$ consisting of vectors of the form $\left(x_{1}, x_{2}, \ldots, x_{n}\right)$, where $x_{i} \neq 0$ for any $i=1, \ldots, n$. We can identify $\mathbb{G}_{\mathrm{m}}^{n}$ with the diagonal maximal torus in $\mathrm{GL}_{n}$. Now

$$
V_{n} / T<\stackrel{\simeq}{\simeq}\left(\mathbb{G}_{\mathrm{m}}\right)^{n} / T \underset{\operatorname{det}}{\simeq} \mathbb{G}_{\mathrm{m}},
$$

where $\mathrm{S}_{n}$ acts on $\mathbb{G}_{\mathrm{m}}$ by $\sigma \cdot t=\operatorname{sign}(\sigma) t$. Thus the kernel of the $H$-action on $V_{n} / T$ is $H \cap \mathrm{A}_{n}$, as claimed.

Proof of Theorem 9.1. We will assume that $\Gamma=\mathrm{SL}_{n}$ and $T$ is the diagonal torus in $\Gamma$. The inequalities

$$
\left\lfloor\frac{n}{p}\right\rfloor \leqslant \operatorname{ed}(N ; p) \leqslant\left\lfloor\frac{n}{p}\right\rfloor+1 ;
$$

are known for every $n$ and $p$; see [Mac11, Section 5.4]. We will write $V_{n}$ for the natural $n$ dimensional representation of $\mathrm{SL}_{n}$ (which we will sometimes restrict to $N$ or subgroups of $N$ ).

(a) Assume that $p$ is an odd prime and $n$ is divisible by $p$. Let $H \simeq(\mathbb{Z} / p \mathbb{Z})^{n / p}$ be the subgroup of $W=N / T \simeq \mathrm{S}_{n}$ generated by the commuting $p$-cycles $\left(\begin{array}{l}1 \\ 2\end{array} p\right),(p+1 p+2 \ldots 2 p), \ldots$, $(n-p+1 \ldots n)$. Since $H$ is a $p$-group, it lies in a Sylow $p$-subgroup $W_{p}$ of $\mathrm{S}_{n}$. Denote the preimage of $H$ in $N$ by $N^{\prime}$. Then $N^{\prime}$ is a subgroup of $N$ of finite index, so

$$
\operatorname{ed}(N ; p) \geqslant \operatorname{ed}\left(N^{\prime} ; p\right)
$$

see [BRV10, Lemma 2.2]. It thus suffices to show that ed $\left(N^{\prime} ; p\right)=n / p+1$.

Claim. $\eta\left(N^{\prime}\right)=n$.

Suppose that the claim is established. Then $V_{n}$ is a $p$-faithful representation of $N^{\prime}$ of minimal dimension. Since $p$ is odd, $H$ lies in the alternating group $\mathrm{A}_{n}$. By Lemma 9.4(a), the stabilizer in general position for the $N^{\prime}$-action on $V$ is isomorphic to $H$. By Theorem 1.2,

$$
\operatorname{ed}\left(N^{\prime} ; p\right)=\operatorname{dim}\left(V_{n}\right)+\operatorname{rank}(H)-\operatorname{dim}\left(N^{\prime}\right)=n+\frac{n}{p}-(n-1)=\frac{n}{p}+1,
$$

and we are done.

To prove the claim, note that $N^{\prime}$ has a faithful representation $V_{n}$ of dimension $n$. Hence, $\eta\left(N^{\prime}\right) \leqslant n$. To prove the opposite inequality, $\eta\left(N^{\prime}\right) \geqslant n$, it suffices to show that

$$
\operatorname{SymRank}(X(T) ; p) \geqslant n
$$

see Lemma 9.3. Here we view $X(T)$ as an $H$-lattice. By definition, $\operatorname{SymRank}(X(T) ; p)$ is the minimal cardinality of a finite $\mathrm{H}$-invariant $p$-spanning subset $\left\{x_{1}, \ldots, x_{d}\right\} \subset X(T)$. The $H$-action on $\left\{x_{1}, \ldots, x_{d}\right\}$ gives rise to a permutation representation $\varphi: H \rightarrow \mathrm{S}_{d}$.

The permutation representation $\varphi$ is necessarily faithful. Indeed, assume the contrary: $1 \neq h$ lies in the kernel of $\varphi$. Then $x_{1}, \ldots, x_{d}$ lie in $X(T)^{h}$. On the other hand, it is easy to see that $X(T)^{h}$ is of infinite index in $X(T)$. Hence, $\left\{x_{1}, \ldots, x_{d}\right\}$ cannot be a $p$-spanning subset of $X(T)$. This contradiction shows that $\varphi$ is faithful.

Now [AG89, Theorem 2.3(b)] tells us that the order of any abelian $p$-subgroup of $\mathrm{S}_{d}$ is at most $p^{d / p}$. In particular, $|H| \leqslant p^{d / p}$. In other words, $p^{n / p} \leqslant p^{d / p}$ or, equivalently, $n \leqslant d$. This completes the proof of (9.4) and thus of the claim and of part (a). 


\section{EXTENSIONS OF FINITE GROUPS BY TORI}

(b) When $p=2$ and $n$ is even, the argument in part (a) does not work as stated because it is no longer true that $H$ lies in the alternating group $\mathrm{A}_{n}$. However, when $n$ is divisible by 4 , we can redefine $H$ as

$$
H_{1} \times \cdots \times H_{n / 4} \hookrightarrow \underbrace{\mathrm{A}_{4} \times \cdots \times \mathrm{A}_{4}}_{(n / 4 \text { times })} \hookrightarrow A_{n},
$$

where $H_{i} \simeq(\mathbb{Z} / 2 \mathbb{Z})^{2}$ is the unique normal subgroup of order 4 in the $i$ th copy of $\mathrm{A}_{4}$. With $H$ defined this way, $H \simeq(Z / 2 \mathbb{Z})^{n / 2}$ is a subgroup of $\mathrm{A}_{n}$, and the rest of the proof of part (a) goes through unchanged.

(c) Write $n=p q+r$, where $1 \leqslant r \leqslant p-1$. The subgroup of $\mathrm{S}_{n}$ consisting of the permutations $\sigma$ such that $\sigma(i)=i$ for any $i>p q$, is naturally identified with $\mathrm{S}_{p q}$. Let $P_{p q}$ be a $p$-Sylow subgroup of $\mathrm{S}_{p q}$, and let $N^{\prime}$ be the preimage of $P_{p q}$ in $N$. Then $\left[N: N^{\prime}\right]=\left[\mathrm{S}_{n}: P_{p q}\right]$ is prime to $p$; hence, it suffices to show that $\operatorname{ed}\left(N^{\prime} ; p\right)=\lfloor n / p\rfloor$. In view of $(9.2)$, it is enough to show that $\operatorname{ed}\left(N^{\prime} ; p\right) \leqslant\lfloor n / p\rfloor$. Since $r \geqslant 1$, as an $N^{\prime}$-representation, $V_{n}$ splits as $k^{p q} \oplus k^{r}$ in the natural way. Let us now write $k^{r}$ as $k^{r-1} \oplus k$ and combine $k^{r-1}$ with $k^{p q}$. This yields a decomposition $V_{n}=k^{n-1} \oplus k$, where the action of $N^{\prime}$ on $k^{n-1}$ is faithful. Now recall that $P_{p q}$ has a faithful $q$-dimensional representation; see, for example, the proof of [MR09, Lemma 4.2]. Denote this representation by $V^{\prime}$. Viewing $V^{\prime}$ as a $q$-dimensional representation of $N^{\prime}$ via the natural projection $N^{\prime} \rightarrow P_{p q}$, we obtain a generically free representation $k^{n-1} \oplus V^{\prime}$ of $N^{\prime}$. Thus

$$
\operatorname{ed}\left(N^{\prime} ; p\right) \leqslant \operatorname{dim}\left(k^{n-1} \oplus V^{\prime}\right)-\operatorname{dim}\left(N^{\prime}\right)=(n-1)+q-(n-1)=q=\left\lfloor\frac{n}{p}\right\rfloor,
$$

as desired.

(d) The argument of part (c) is valid for any prime. In particular, if $p=2$, it proves part (d) in the case where $n$ is odd. Thus we may assume without loss of generality that $n \equiv 2(\bmod 4)$. Let $N^{\prime}$ be the preimage of $P_{n}$ in $N$, where $P_{n}$ is a Sylow 2-subgroup of $\mathrm{S}_{n}$. Then the index $\left[N: N^{\prime}\right]=\left[\mathrm{S}_{n}: P_{n}\right]$ is finite and odd; hence, $\operatorname{ed}(N ; 2)=\operatorname{ed}\left(N^{\prime} ; 2\right)$. In view of $(9.2)$, it suffices to show that $\operatorname{ed}\left(N^{\prime} ; 2\right) \leqslant n / 2$.

Since $n \equiv 2(\bmod 4)$, we have $P_{n}=P_{n-2} \times P_{2}$, where $P_{2} \simeq \mathrm{S}_{2}$ is the subgroup of $\mathrm{S}_{n}$ of order 2 generated by the 2-cycle $(n-1, n)$. Let $V^{\prime}$ be a faithful representation of $P_{n-2}$ of dimension $(n-2) / 2$. We may view $V^{\prime}$ as a representation of $N^{\prime}$ via the projection $N^{\prime} \rightarrow P_{n} \rightarrow P_{n-2}$.

Claim. The action of $N^{\prime}$ on $V_{n} \oplus V^{\prime}$ is generically free.

If this claim is established, then

$$
\operatorname{ed}\left(N^{\prime}\right) \leqslant \operatorname{dim}\left(V_{n} \oplus V^{\prime}\right)-\operatorname{dim}\left(N^{\prime}\right)=n+\frac{n-2}{2}-(n-1)=\frac{n}{2},
$$

and we are done.

To prove the claim, let $S$ the stabilizer in general position for the action of $N^{\prime}$ on $V_{n}$. Denote the natural projection $N^{\prime} \rightarrow P_{n}$ by $\pi$. By Lemma 9.4(a), we have $S \cap T=1$. In other words, $\pi$ is an isomorphism between $S$ and $\pi(S)$. Since $P_{n}=P_{n-2} \times P_{2}$, the kernel of the $P_{n}$-action on $V^{\prime}$ is $P_{2}$. It now suffices to show that $S$ acts faithfully on $V^{\prime}$, that is, $\pi(S) \cap P_{2}=1$.

By Lemma 9.4, we have $\pi(S) \subset \mathrm{A}_{n}$; that is, every permutation in $\pi(S)$ is even. On the other hand, the non-trivial element of $P_{2}$, namely the transposition $(n-1, n)$, is odd. This shows that $\pi(S) \cap P_{2}=1$, as desired.

Proof of Theorem 9.2. By [Mac11, Section 5.7], we have ed $(N ; 2) \leqslant 4 n$. Thus it suffices to show that $\operatorname{ed}(N ; 2) \geqslant 4 n$. 


\section{Z. Reichstein AND F. Scavia}

Recall that a split maximal torus $T$ of $\mathrm{SO}_{4 n}$ is isomorphic to $\left(\mathbb{G}_{\mathrm{m}}\right)^{2 n}$ and the Weyl group $W$ is a semi-direct product $A \rtimes \mathrm{S}_{2 n}$. Here $A \simeq(\mathbb{Z} / 2 \mathbb{Z})^{2 n-1}$ is the multiplicative group of $2 n$-tuples $\epsilon=\left(\epsilon_{1}, \ldots, \epsilon_{2 n}\right)$, where each $\epsilon_{i}$ is \pm 1 and $\epsilon_{1} \epsilon_{2} \cdots \epsilon_{2 n}=1$. The symmetric group $\mathrm{S}_{2 n}$ acts on $A$ by permuting $\epsilon_{1}, \ldots, \epsilon_{2 n}$. The action of $W$ on $\left(t_{1}, \ldots, t_{2 n}\right) \in T$ is as follows: $\mathrm{S}_{2 n}$ permutes $t_{1}, \ldots, t_{2 n}$, and $\epsilon$ takes each $t_{i}$ to $t_{i}^{\epsilon_{i}}$. The normalizer $N$ of $T$ in $\mathrm{SO}_{4 n}$ is the semidirect product of $T$ and $W$ with respect to this action.

Let $H$ be the subgroup of $W$ generated by elements $\left(\epsilon_{1}, \ldots, \epsilon_{2 n}\right) \in A$ with $\epsilon_{1}=\epsilon_{2}, \epsilon_{3}=\epsilon_{4}$, $\ldots, \epsilon_{2 n-1}=\epsilon_{2 n}$ and the $n$ disjoint 2 -cycles $(1,2),(3,4), \ldots,(2 n-1,2 n)$ in $\mathrm{S}_{2 n}$. It is easy to see that these generators are of order 2 and commute with each other, so that $H \simeq(\mathbb{Z} / 2 \mathbb{Z})^{n}$. Let $N^{\prime}$ be the preimage of $H$ in $N$.

Note that $H$ arises as a stabilizer in general position of the natural $4 n$-representation $V_{4 n}$ of $N$ (restricted from $\left.\mathrm{SO}_{4 n}\right)$. Here $\left(t_{1}, \ldots, t_{2 n}\right) \in T$ acts on $\left(x_{1}, \ldots, x_{2 n}, y_{1}, \ldots, y_{2 n}\right) \in V_{4 n}$ by $x_{i} \mapsto t_{i} x_{i}$ and $y_{i} \mapsto t_{i}^{-1} y_{i}$ for each $i$. The symmetric group $\mathrm{S}_{2 n}$ simultaneously permutes $x_{1}, \ldots, x_{2 n}$ and $y_{1}, \ldots, y_{2 n}$; the $2 n$-tuple $\epsilon \in A$ leaves $x_{i}$ and $y_{i}$ invariant if $\epsilon_{i}=1$ and switches them if $\epsilon_{i}=-1$.

Note that $N^{\prime}$ is a subgroup of finite index in $N$. Hence, ed $(N ; 2) \geqslant \operatorname{ed}\left(N^{\prime} ; 2\right)$, and it suffices to show that $\operatorname{ed}\left(N^{\prime} ; 2\right) \geqslant 4 n$.

Claim. $\eta\left(N^{\prime}\right)=4 n$.

Suppose for a moment that the claim is established. Then $V_{4 n}$ is a 2-faithful representation of $N^{\prime}$ of minimal dimension. As we mentioned above, a stabilizer in general position for this representation is isomorphic to $H$. By Theorem 1.2,

$$
\operatorname{ed}\left(N^{\prime} ; 2\right)=\operatorname{dim}\left(V_{4 n}\right)+\operatorname{rank}(H)-\operatorname{dim}\left(N^{\prime}\right)=4 n+2 n-2 n=4 n,
$$

and we are done.

To prove the claim, note that $\eta\left(N^{\prime}\right) \leqslant 4 n$ since $N^{\prime}$ has a faithful representation $V_{4 n}$ of dimension $4 n$. By Lemma 9.4, in order to establish the opposite inequality, $\eta\left(N^{\prime}\right) \geqslant 4 n$, it suffices to show that $\operatorname{SymRank}(X(T) ; 2) \geqslant 4 n$. To prove this last inequality, we will use the same argument as in the proof of Theorem 9.1(a). Recall that $\operatorname{SymRank}(X(T) ; 2)$ is the minimal size of an $H$-invariant 2-generating set $x_{1}, \ldots, x_{d}$ of $X(T)$. The $H$-action on $x_{1}, \ldots, x_{d}$ induces a permutation representation $\varphi: H \rightarrow \mathrm{S}_{d}$. Once again, this representation has to be faithful. By [AG89, Theorem 2.3(b)], we have $|H| \leqslant 2^{d / 2}$. In other words, $2^{2 n} \leqslant 2^{d / 2}$ or, equivalently, $d \geqslant 4 n$, as claimed.

\section{ACKNOWLEDGEMENTS}

We are grateful to Jerome Lefebvre, Mathieu Huruguen, and Mikhail Borovoi for stimulating discussions related to Conjecture 1.1 and to the anonymous referee for constructive comments.

\section{REFERENCES}

AG89 M. Aschbacher and R. M. Guralnick, On abelian quotients of primitive groups, Proc. Amer. Math. Soc. 107 (1989), no. 1, 89-95; doi:10.2307/2048039.

Art86 M. Artin, Néron models, Arithmetic Geometry (Storrs, Conn., 1984) (Springer, New York, 1986), 213-230; doi:10.1007/978-1-4613-8655-1_8.

Bou89 N. Bourbaki, Commutative algebra. Chapters 1-7, Elements of Mathematics (Springer-Verlag, Berlin, 1989). 


\section{EXTENSIONS OF FINITE GROUPS BY TORI}

BR85 P. Bardsley and R. W. Richardson, Étale slices for algebraic transformation groups in characteristic p, Proc. London Math. Soc. 51 (1985), no. 2, 295-317; doi:10.1112/plms/s351.2.295.

BRV10 P. Brosnan, Z. Reichstein, and A. Vistoli, Essential dimension, spinor groups, and quadratic forms, Ann. of Math. 171 (2010), no. 1, 533-544; doi:10.4007/annals.2010.171.533.

BRV18 _ Essential dimension in mixed characteristic, Doc. Math. 23 (2018), 1587-1600; doi: $10.25537 / \mathrm{dm} .2018 \mathrm{v} 23.1587-1600$.

CR62 C.W. Curtis and I. Reiner, Representation theory of finite groups and associative algebras, Pure Appl. Math., vol. 11 (Interscience Publ., New York - London, 1962).

GR09 P. Gille and Z. Reichstein, A lower bound on the essential dimension of a connected linear group, Comment. Math. Helv. 84 (2009), no. 1, 189-212; doi:10.4171/CMH/158.

Jan04 J. C. Jantzen, Nilpotent orbits in representation theory, Lie Theory, Progr. Math., vol. 228 (Birkhäuser Boston, Boston, MA, 2004), 1-211; doi:10.1007/978-0-8176-8192-0_1.

KM08 N. A. Karpenko and A.S. Merkurjev, Essential dimension of finite p-groups, Invent. Math. 172 (2008), no. 3, 491-508; doi:10.1007/s00222-007-0106-6.

Lan02 S. Lang, Algebra, 3rd ed., Grad. Texts in Math., vol. 211 (Springer-Verlag, New York, 2002); doi:10.1007/978-1-4613-0041-0.

LMMR13a R. Lötscher, M. MacDonald, A. Meyer, and Z. Reichstein, Essential dimension of algebraic tori, J. reine angew. Math. 677 (2013), 1-13; doi:10.1515/crelle.2012.010.

LMMR13b _ Essential p-dimension of algebraic groups whose connected component is a torus, Algebra Number Theory 7 (2013), no. 8, 1817-1840; doi:10.2140/ant.2013.7.1817.

Mac11 M. L. MacDonald, Essential p-dimension of the normalizer of a maximal torus, Transform. Groups 16 (2011), no. 4, 1143-1171; doi:10.1007/s00031-011-9157-2.

Mar15 B. Martin, Generic stabilisers for actions of reductive groups, Pacific J. Math. 279 (2015), no. 1-2, 397-422; doi:10.2140/pjm.2015.279.397.

Mer13 A.S. Merkurjev, Essential dimension: a survey, Transform. Groups 18 (2013), no. 2, 415-481; doi:10.1007/s00031-013-9216-y.

MR09 A. Meyer and Z. Reichstein, The essential dimension of the normalizer of a maximal torus in the projective linear group, Algebra Number Theory 3 (2009), no. 4, 467-487; doi:10.2140/ ant.2009.3.467.

PV94 V.L. Popov and E. B. Vinberg, Invariant theory, Algebraic Geometry IV, Encyclopaedia Math. Sci., vol. 55 (Springer, Berlin - Heidelberg, 1994), 123-278; doi:10.1007/978-3-66203073-8_2.

Rei10 Z. Reichstein, Essential dimension, Proceedings of the ICM (2010), vol. 2 (Hindustan Book Agency, New Delhi, 2010), 162-188; doi:10.1142/9789814324359_0045.

Ric72 R.W. Richardson, Jr., Deformations of Lie subgroups and the variation of isotropy subgroups, Acta Math. 129 (1972), 35-73; doi:10.1007/BF02392213.

RY00 Z. Reichstein and B. Youssin, Essential dimensions of algebraic groups and a resolution theorem for $G$-varieties (with an appendix by János Kollár and Endre Szabó), Canad. J. Math. 52 (2000), no. 5, 1018-1056; doi:10.4153/CJM-2000-043-5.

Ser97 J.-P. Serre, Galois cohomology (Springer-Verlag, Berlin, 1997); doi:10.1007/978-3-64259141-9.

Zinovy Reichstein reichst@math.ubc.ca

Department of Mathematics, University of British Columbia, Vancouver, BC V6T 1Z2, Canada

Federico Scavia scavia@math.ubc.ca

Department of Mathematics, University of British Columbia, Vancouver, BC V6T 1Z2, Canada 